\title{
Evaluación de impacto de políticas activas de empleo para colectivos de difícil inserción laboral*
}

\section{Active labor market policies for workers with difficult job placement experiences: an impact evaluation}

\author{
Yolanda Fátima Rebollo-Sanz \\ Universidad Pablo de Olavide \\ José Ignacio García Pérez \\ Universidad Pablo de Olavide y FEDEA
}

\begin{abstract}
Resumen
En este trabajo se realiza un ejercicio de evaluación de impacto de una política activa típicamente dirigida a parados de difícil inserción laboral. Se trata de las denominadas iniciativas locales de empleo y, concretamente, de dos medidas desarrolladas en Andalucía durante los años 2016-2018: los programas Emple@Joven y Emple@30+. Utilizando métodos de emparejamiento se obtiene que el programa Emple@Joven, dirigido a parados menores de 30 años, no aumenta los niveles de empleabilidad para los beneficiarios del programa y que el programa Emple@30+, dirigido a parados mayores de 30 años, solo favorece la empleabilidad a los parados sin experiencia laboral o aquellos que han estado fuera del mercado de trabajo durante más de 24 meses. Por tanto, estos programas, que ofrecen una experiencia laboral de entre 3 y 6 meses, solo parecen ser efectivos para colectivos muy determinados con especial dificultad para acceder por sí mismos al mercado de trabajo. Este ejercicio de evaluación apunta la necesidad de perfilar mejor a los beneficiarios de las políticas activas de empleo y ofrecer a estos solo las que se estime puedan ofrecer un mayor impacto sobre su nivel de empleabilidad, ayudándoles a una mejor y más estable inserción en el mercado de trabajo.
\end{abstract}

Palabras clave: evaluación de políticas activas de empleo, iniciativas locales de empleo, propensity score matching.

Clasificación JEL: J24, J68, H53.

\section{Abstract}

In this paper, an impact assessment exercise of an active labour market policy aimed at hard-toplace unemployed is conducted. These are the so-called local employment initiatives and in particular, two measures developed in Andalusia during the years 2016-2018: the Emple@Joven and Emple@30+ programs. Using matching methods, it is found that the Emple@Joven program, aimed at unemployed people under the age of 30, did not increase the employment levels for the beneficiaries of the program and that the Emple@30+ program, aimed at unemployed people over the age of 30, only significantly helped the unemployed people without working experience or those who had been out of the labour market for more than 24 months. Therefore, apparently, these measures that provided a working experience between 3 and 6 months, only seemed to be effective for very specific groups with special difficulty in accessing the labour market on their own. These results, thus, point to the need of a better ex-ante profiling of active employment policies' recipients, offering them only to those who are expected to gain the most in terms of their level of employability, offering them a better and more stable insertion in the labour market.

Keywords: active employment policy evaluation, local employment initiatives, propensity score matching.

* Los autores agradecen a la Consejería de Empleo, Formación y Trabajo Autónomo de la Junta de Andalucía la cesión de los datos necesarios para poder realizar este trabajo. También agradecen la financiación recibida de la Agencia Estatal de Investigación (Ministerio de Ciencia e Innovación), proyecto PID2019-104452RB-I00 y del Fondo Europeo de Desarrollo Regional y la Consejería de Transformación Económica, Industria, Conocimiento y Universidades de la Junta de Andalucía, proyectos P18- RT-2135 y CV20-35470. Cualquier error que pudiera incluir el texto es de la exclusiva responsabilidad de sus autores. 


\section{Introducción}

Es común encontrar informes y documentos de política económica que califican las políticas activas de empleo (PAE) como una herramienta fundamental en la lucha contra el desempleo. De hecho, el papel de estas políticas ha sido y es crucial en Europa como estrategia para mejorar la empleabilidad. Desde que se aprobó por primera vez la Estrategia Europea para el Empleo (EEE) en 1997, han aumentado considerablemente los presupuestos para los programas dirigidos al fomento del empleo de colectivos específicos (jóvenes, parados de larga duración, mujeres, personas en riesgo de exclusión social, etc.). Sin embargo, en pocas ocasiones dichas recomendaciones están sustentadas por un conocimiento claro del impacto de cada una de estas políticas sobre el acceso al empleo o los ingresos futuros de los beneficiarios potenciales. Si bien es cierto que a nivel internacional se cuenta cada vez con más evaluaciones del impacto de las PAE (hay estudios que sistematizan los resultados con un metaanálisis, Card, Kluve \& Weber, 2010; Kluve, 2010; o Card et al., 2018), en España se sigue padeciendo de una escasez de evaluaciones de las distintas PAE en general (algunas excepciones son García-Pérez \& Rebollo-Sanz, 2009a, 2009b; Cueto \& Mato, 2009; Arranz et al., 2013; De la Rica, 2015). Y aún más extrema es la escasez de ejercicios de evaluación de impacto de las iniciativas locales de empleo, objeto del presente artículo.

En este sentido, este trabajo presenta los resultados de un ejercicio de evaluación de impacto de las iniciativas locales de empleo que se ejecutaron en la Comunidad Autónoma de Andalucía durante los años 2016-2018. Esta política se desarrolló bajo el nombre de Proyectos de Cooperación Social y Comunitaria y tenía como objetivo ofrecer experiencias de empleo temporales a sus beneficiarios, prioritariamente individuos parados con dificultades de inserción laboral, de cara a mejorar su empleabilidad futura y su completa inserción en el mercado de trabajo.

Este tipo de actuaciones han sido muy usadas en España durante los últimos 10-15 años, pero fue a raíz de la última gran crisis económica cuando surgieron con especial énfasis este tipo de medidas en distintas comunidades autónomas. En efecto, en el año 2008 comenzó lo que vino a llamarse la Gran Depresión. Cinco años después, la tasa de paro era todavía muy elevada, con niveles que superaban, en el año 2015, el $20 \%$ en el conjunto de España y el $29 \%$ en Andalucía. La tasa de paro juvenil era mucho más abultada, con niveles que superaban el $45 \%$ en España y el $55 \%$ en Andalucía. Pero la manifestación más dramática de la dureza de esta crisis fue el desempleo de larga duración. En 2015, tanto en el conjunto de España como en Andalucía, más de la mitad de los desempleados llevaban más de 12 meses sin al menos un día de trabajo y, por tanto, con una desconexión del mundo laboral muy prolongada.

Con la finalidad de hacer frente a las consecuencias de esta grave crisis, la mayoría de las regiones españolas pusieron en marcha medidas de empleo y acciones urgentes para tratar de activar a los desempleados, especialmente en aquellos sectores de población con más dificultades de acceso al empleo o con más barreras para la 
inserción laboral. Uno de los tipos de PAE que se desarrollaron en algunas CC. AA., tales como Madrid, Cataluña, Aragón, Navarra, Extremadura, Canarias y Andalucía, fueron las iniciativas locales de empleo. En el caso concreto de la Comunidad Autónoma de Andalucía, el Gobierno regional dictó el Decreto-ley 2/2015, de 3 de marzo, de medidas urgentes para favorecer la inserción laboral, la estabilidad en el empleo, el retorno del talento y el fomento del trabajo autónomo, del que se derivaron los siguientes programas ${ }^{1}$ : Emple@Joven y programa Emple@30+. Ambos programas ponían énfasis en la creación de empleo en la Comunidad Autónoma de Andalucía, así como en la mejora de la empleabilidad de determinados colectivos con dificultades de acceso al empleo. Una característica distintiva de estos programas de empleo público que favoreció su ejecución frente a otro tipo de PAE, fue el alto nivel de descentralización en la aplicación del programa. Las ofertas de empleo se generan a nivel municipal y esto permitía que estas se adaptasen a las necesidades locales de la población desempleada.

Los objetivos fundamentales de este trabajo se concretan en, primero, caracterizar a los beneficiarios de este programa de Cooperación Social y Comunitaria desarrollado por Andalucía durante los años 2016-2018 y comprobar si, efectivamente, se ha beneficiado a los colectivos más vulnerables y, por ende, con mayores dificultades para su inserción laboral. Segundo, comprobar la empleabilidad posterior de los participantes y su relación de causalidad con ser beneficiarios de dicho programa. Nuestro objetivo final es ofrecer unos resultados que sirvan de referencia para propiciar un debate sobre el diseño de este tipo de políticas activas de empleo. De este debate deberían surgir propuestas de mejora en el diseño de nuevos programas con objetivos similares.

La evaluación de impacto es un método de gran utilidad para responder a la pregunta de en qué medida el cambio en una variable determinada, que mide el resultado de una intervención, es debido a la intervención analizada. Una rigurosa cuantificación de los impactos de los programas o intervenciones implica la elaboración de contrafactuales que permitan identificar y/o estimar qué habría sucedido en ausencia de una intervención específica. El ejercicio de evaluación lo realizaremos aplicando modelos de emparejamiento (propensity score matching, PSM). Disponemos de una base de datos de demandantes de empleo proporcionada por el Servicio Andaluz de Empleo (SAE). En dicha base de datos, disponemos de la información necesaria para construir los grupos de tratamiento y control. Los primeros son los participantes en el programa y los segundos son demandantes de empleo que cumplen los requisitos para participar, pero no lo han hecho.

Nuestros resultados fundamentales indican que las iniciativas locales de empleo no han favorecido, en media, un aumento en la probabilidad de salir del paro al empleo. Sin embargo, sí encontramos que para algunos colectivos existen efectos positivos, aunque de pequeña cuantía. Estos colectivos son: demandantes de empleo

${ }^{1}$ Anteriormente, se había ejecutado un programa parecido: Emple@ Joven e Iniciativa @mprende+ (Decreto-ley 6/2014, de 29 de abril) y programa Emple@30+ (Decreto-ley 9/2014, de 15 de julio). 
mayores de 30 años sin experiencia laboral o con duraciones de paro superiores a los dos años. Por tanto, parece que esta iniciativa puede estar más recomendada para dotar de experiencia laboral a los desempleados, o bien que no hayan trabajado nunca, o bien para parados de larga duración que necesitan reconectar con el sector productivo.

La estructura del resto del artículo es la siguiente. En la segunda sección se presenta una revisión de la literatura empírica. En la tercera sección se describe el programa objeto de análisis. En la cuarta y quinta sección se presentan la metodología empleada y la descripción de la fuente de información y variables utilizadas. En la sexta sección se comentan los resultados encontrados y se finaliza con las conclusiones.

\section{Revisión literatura empírica}

Es bastante escasa la literatura empírica que evalúa iniciativas de creación directa de empleo público dirigidas a colectivos con dificultades de inserción laboral. Esto es así tanto para España como para otros países europeos. La mayoría de la literatura empírica existente en España se centra en la evaluación de otro tipo de PAE como programas de formación (Blázquez, Herrarte \& Sáez, 2019²; Cueto \& Mato, 2009³; Arellano, 2010 4 ; INAEM, 2015 ${ }^{5}$ ) o subsidios salariales a la contratación (García-Pérez \& Rebollo-Sanz, 2009a), con el objetivo de conocer su impacto sobre los niveles de empleabilidad o estabilidad laboral de los beneficiarios de los programas ${ }^{6}$. Generalmente, a su vez, este tipo de trabajos evalúan los efectos de corto plazo y en pocas ocasiones los efectos de medio plazo relacionados con la calidad y la duración del nuevo empleo (una excepción es García-Pérez \& Rebollo-Sanz, 2009b).

${ }^{2}$ Estos autores analizan la influencia de programas de formación y asistencia en la búsqueda de empleo en la Comunidad de Madrid sobre la probabilidad de conseguir un empleo para los parados de larga duración (PLD). Los resultados del estudio mostraban que la participación en ambos programas ejercía un efecto positivo en la empleabilidad de los beneficiarios, siendo el efecto de la participación en programas de formación mucho más intenso que el de asistencia a la búsqueda de empleo, tanto en términos de empleabilidad como de calidad en el empleo.

3 En este trabajo se estudió el efecto de un programa de formación regional voluntario sobre la probabilidad de empleo y encontraron que la formación aumentaba las probabilidades de empleo entre un $8 \%$ y un $9 \%$.

${ }^{4}$ En él se evalúa el efecto causal de los cursos de formación sobre la duración del desempleo constatando que los cursos de nivel medio redujeron el desempleo entre los trabajadores que lo recibieron.

${ }^{5}$ En 2015 se realizó una evaluación de las PAE llevadas a cabo por el Instituto Aragonés de Empleo (INAEM). En este caso concreto, se analizaron los efectos individuales de tres tipos de medidas (promoción, intermediación y formación). Respecto a las políticas de promoción de empleo se obtuvo que en el primer año un individuo beneficiario de la ayuda trabajaba más tiempo que otro similar que no ha participado. En cuanto a los PLD, grupo objetivo de estas políticas, obtienen un diferencial relativamente más elevado (hasta el tercer mes) que el del resto de desempleados. Respecto a las políticas de formación, los autores encuentran que, en media, estas contribuyen positivamente tanto a la probabilidad de salida del paro (evaluada tras 12 meses) como a la estabilidad del nuevo empleo. No obstante, este efecto positivo no se encuentra para los PLD.

${ }^{6}$ Se han seleccionado algunos de los artículos publicados en los últimos 15 años. 
Una referencia fundamental en la literatura empírica relacionada con la evaluación de impacto de las PAE son los metaanálisis de Card, Kluve y Weber (2010, 2018) y Kluve (2010). En estos trabajos se concluye que los programas de creación directa de empleo por parte del sector público son menos eficaces que otro tipo de medidas, como los programas de formación o los de asistencia para la búsqueda de empleo en la mejora de la empleabilidad de los beneficiarios de dichos programas. En concreto, estos metaanálisis constatan que, en media, los programas de empleo público ofrecen malos rendimientos en términos de la empleabilidad de sus beneficiarios. Uno de los mecanismos que pueden generar estos efectos negativos es la duración prolongada de los empleos subvencionados (en la literatura, este efecto se denomina lock in effect). La idea fundamental de este enfoque es que el individuo queda «atrapado» en el empleo público cuando la duración del programa es prolongada. A medida que permanecen en el empleo subvencionado, los beneficiarios van recibiendo menos ofertas de trabajo o, si las reciben, las van a rechazar con una alta probabilidad. Esto puede terminar perjudicando las perspectivas laborales al futuro del participante, a pesar de haber ganado algo de experiencia laboral por haber participado en el programa.

Otros trabajos que también hacen evaluación de impacto y llegan a conclusiones similares son los de Kuddo (2009) y Escudero (2016, 2019). Kuddo (2009) revisa las PAE en países de Europa del Este y Asia Central y destaca sus efectos favorables sobre la reducción de la tasa de pobreza en el corto plazo, aunque no encuentra efectos positivos sobre mejoras en el nivel de empleabilidad de los colectivos beneficiarios del programa ${ }^{7}$. En Escudero et al. (2019) se analizan los resultados de evaluaciones de impacto de diferentes PAE en América Latina y el Caribe. La evaluación del programa Construyendo Perú llevada a cabo por Escudero (2016) muestra que la iniciativa permitió generar empleo y reducir la inactividad de los grupos beneficiarios, pero a costa de asumir empleos de menor calidad. La evaluación del programa PLANE implementado en Bolivia muestra que, en el contexto de alta rigidez del mercado laboral en el que se desarrolló la política, esta no tuvo impacto en la probabilidad de empleo posterior a la intervención (Hernani-Limarino et al., 2011).

Por otra parte, también hay trabajos que apuntan mejoras en las habilidades o en la empleabilidad de los individuos beneficiarios de programas de creación directa de empleo público. Por ejemplo, Roy y Wong (2000) revisa la relación coste-efectividad de programas de creación directa de empleo llevados a cabo por el Gobierno canadiense en las décadas de los años ochenta y noventa del pasado siglo y concluye que este tipo de programas es adecuado para colectivos desfavorecidos porque favorece el desarrollo de habilidades. Hohmeyer y Wolff (2010) evalúan el impacto de tres programas de creación de empleo directo dirigidos a individuos con dificultades de

${ }^{7}$ Kuddo (2009) destaca el caso de un programa masivo público implantado en Bulgaria en 2002, From Social Assistance to Employment, dirigido a grupos desfavorecidos, como aquellos con niveles de educación bajo, desempleados de larga duración y beneficiarios de asistencia social. Los resultados del análisis de impacto indicaban que solo el $8 \%$ de los participantes de estos programas encontraron empleo una vez finalizado, mientras que para el grupo de control este porcentaje era del $16 \%$. 
inserción laboral (beneficiarios de la asistencia social en Alemania) que tuvieron lugar en Alemania entre 2005 y 2008. Los autores concluyen que estos programas contribuyeron a un mejor desempeño laboral entre los participantes.

Para el contexto de España, la literatura empírica centrada en la evaluación de impacto de programas de empleo público es notoriamente escasa, a pesar de que, en los últimos años y tras la Gran Depresión, existen multitud de iniciativas locales de empleo. Entre las excepciones se encuentra el trabajo de Ramos, Surinach y Artís (2009). Estos autores estudian la eficacia de una amplia gama de PAE aplicadas en Cataluña en los años previos a la Gran Depresión (2007-2008). Entre las políticas evaluadas se encuentran las acciones de fomento al empleo en el ámbito local como los Planes de Empleo ${ }^{8}$. Para la mayoría de los programas analizados los autores encuentran un aumento en la probabilidad de empleo de los participantes dos años después del programa, sobre todo para los beneficiarios de planes públicos de empleo 9 , apoyo al empleo personalizado y formación profesional.

En resumen, de la revisión de la literatura empírica se puede concluir que existen aún pocos estudios que evalúen la efectividad de las iniciativas locales de empleo. Aún más escasos son los estudios de este tipo de PAE para el caso español. La evidencia encontrada apunta que no suelen tener un marcado efecto positivo en términos de empleabilidad, sobre todo los relacionados con la creación de empleo público. No obstante, la mayoría de los trabajos de evaluación de impacto de las PAE también apuntan la existencia de una gran heterogeneidad en la efectividad de estos programas dependiendo del colectivo al que van dirigidos. Esta gran heterogeneidad de resultados ha puesto de manifiesto la necesidad de que cada programa de empleo público sea diseñado y evaluado en el contexto concreto -mercado de trabajo local- y para el colectivo específico para el que está dirigido (Gerfin \& Lechner, 2002; Card, Kluve \& Weber, 2018). De hecho, en la actualidad, y a medida que proliferan los trabajos de evaluación causal de las PAE, se considera que un buen diseño de una PAE debe ser aquel que sea multidimensional (incluya formación, asistencia en el proceso de búsqueda de empleo, etc.), flexible y se adapte a las necesidades específicas de cada beneficiario potencial. Es decir, un buen diseño de PAE debe llevar asociado un trabajo previo de perfilado que permita identificar a nivel individual las posibilidades de empleabilidad del desempleado, así como sus necesidades de asistencia en la inserción laboral (Felgueroso, García-Pérez \& Jiménez, 2018; Rebollo-Sanz, 2018).

Tampoco los análisis de impacto suelen tener en cuenta otro tipo de efectos relacionados con la incidencia de las PAE sobre la tasa de actividad y/o la tasa de

${ }^{8}$ Programas de contratación laboral de personas que se encuentran en situación de paro para la realización de trabajos de interés público y social en el ámbito local (limpieza, mantenimiento y rehabilitación de edificios municipales, apoyo administrativo y de atención a la ciudadanía, entre otros).

${ }^{9}$ En cuanto al efecto de los diferentes programas, este varía entre el 17,9\% de los Planes de Ocupación y el $4,4 \%$ de las Acciones Integradas (efecto positivo y significativo, para el caso de Planes de Empleo). Para los participantes en los Planes del Empleo, el 63,39\% es el porcentaje de individuos que tenían trabajo a finales de 2007/comienzos de 2008. Del análisis del contrafactual se extrae que si esos individuos no hubiesen participado en dicha iniciativa el porcentaje se reduciría al 45,49\% (caída bastante elevada, no se analizan posibles causas en el trabajo). 
pobreza. Nótese que un potencial objetivo de las PAE dirigidas a los colectivos con especiales dificultades de inserción laboral es también reducir la incidencia del denominado «efecto del trabajador desanimado», esto es, evitar el flujo de salida del paro a la inactividad ${ }^{10}$.

\section{Descripción del programa: las iniciativas locales de empleo}

Las PAE se entienden como el conjunto de servicios y programas de orientación, empleo y formación que tienen como objetivo mejorar las posibilidades de acceso al empleo de los desempleados, el mantenimiento del empleo de los que ya lo tienen, así como fomentar el emprendimiento. Son, por tanto, un conjunto de medidas e iniciativas que buscan mejorar el funcionamiento del mercado laboral mediante instrumentos que ayuden a la creación y el mantenimiento del empleo. Entre los distintos tipos de PAE, las que se evalúan en este trabajo se engloban dentro de las medidas de creación directa de empleo en el sector público (i.e. empleo en las distintas Administraciones públicas o empresas que producen bienes o servicios públicos). Dichos programas, en general, van dirigidos a personas con dificultades de inserción laboral con el fin de mantenerlos en contacto con el mercado de trabajo.

Concretamente, la Ley andaluza 2/2015, de 29 de diciembre, de medidas urgentes para favorecer la inserción laboral, la estabilidad en el empleo, el retorno del talento y el fomento del trabajo autónomo, regula un conjunto de programas cuyo objetivo principal es dar respuesta a las necesidades urgentes de empleo de la población desempleada andaluza. Dentro de estos programas se incluía la denominada Iniciativa Cooperación Social y Comunitaria Emple@Joven y Emple@30+, cuya dotación presupuestaria fue de 250 millones de euros ${ }^{11}$. El programa Emple@Joven estaba dirigido a jóvenes de entre 16-29 años y el programa Emple@30+ a personas de entre 30 y 65 años $^{12}$. Estos programas tenían por objeto mejorar la empleabilidad de las personas demandantes de empleo que se encontraban con dificultades de acceso al mismo, posibilitando la adquisición de competencias profesionales a través de nuevas experiencias de empleo.

${ }^{10}$ Este efecto hace referencia al desaliento de las personas que desean trabajar y no encuentran un empleo en periodos de crisis. Este efecto suele estar relacionado con el estancamiento de la actividad económica y las negativas perspectivas en materia de creación de empleo y se agudiza a medida que el individuo permanece desempleado y sus posibilidades de empleo se reducen.

${ }_{11}$ Otras medidas incluidas en la Ley 2/2015, de 29 de diciembre, de medidas urgentes para favorecer la inserción laboral, la estabilidad en el empleo, el retorno del talento y el fomento del trabajo autónomo son: i) Becas para el desarrollo de prácticas profesionales en empresas, con una dotación de dos millones de euros. ii) Ayudas para la contratación de personas titulares del Bono de Empleo Joven, con una dotación de 70 millones de euros. iii) Iniciativa de Proyectos de Interés General y Social generadores de empleo, con una dotación de 15 millones de euros. iv) Incentivos a la contratación indefinida de personas jóvenes, con una dotación de casi 48 millones de euros. v) Ayudas al trabajo autónomo, con una dotación de 17 millones de euros. Las iniciativas recogidas en esta ley están cofinanciadas con Fondo Social Europeo.

${ }^{12}$ La dotación para cada programa fue de 150 millones para la contratación de los jóvenes entre 18 y 29 años y 100 millones para la contratación del colectivo de más de 30 años. 
En este tipo de programas, las ayudas eran concedidas a los ayuntamientos para la contratación de personas desempleadas residentes en el municipio. Por su parte, las personas destinatarias finales de la iniciativa eran el conjunto de demandantes de empleo no ocupados que reunían una serie de requisitos que comentaremos más adelante. Esta línea de ayudas era gestionada en régimen de concurrencia no competitiva, por lo que los ayuntamientos solo tenían que presentar la solicitud en los términos especificados en la convocatoria para que esta se resolviese favorablemente. El importe de la ayuda consistía en una cantidad a tanto alzado que se determinaba atendiendo a la duración del contrato y al grupo de cotización a la Seguridad Social del individuo contratado ${ }^{13}$. La cuantía máxima de la ayuda a percibir por los municipios se determinaba en función del nivel de desempleo y la población de cada municipio, distinguiendo los límites de edad antes mencionados.

El número total de individuos beneficiarios de estas iniciativas locales de empleo dependían, en primera instancia, de las solicitudes de los ayuntamientos, puesto que sobre ellos recaía la responsabilidad de presentar una oferta de empleo ante el Servicio Andaluz de Empleo (SAE). Este aspecto del diseño del programa (la oferta de trabajo la diseña el ayuntamiento), se ha considerado una característica distintiva y ventajosa de las iniciativas locales de empleo. Esto es así porque favorecía que la oferta de empleo creada por el programa estuviese ligada a las necesidades de empleo local tanto desde el punto de vista de la demanda (tipo de vacantes locales), como desde el punto de vista de la oferta (perfiles de desempleados del municipio). Una vez presentada la oferta por parte del ayuntamiento, el SAE debía tratar de proporcionar tres personas candidatas adecuadas y disponibles en base al perfil solicitado. En caso de que hubiese más de tres participantes potenciales, la convocatoria establecía un orden de prelación según los siguientes parámetros: $i$ ) personas beneficiarias del Ingreso Mínimo de Solidaridad (IMS, en adelante); $i i)$ desempleados de larga duración (PLD) que hayan agotado la prestación por desempleo de nivel contributivo o asistencial en los últimos doce meses; iii) PLD en general; iv) personas desempleadas en general. Por tanto, el programa articulaba la contratación en base a una definición de vulnerabilidad laboral sustentada en las variables renta (a través de la percepción o no del IMS) y duración del desempleo (a través de la pertenencia o no al colectivo de PLD) $)^{14}$.

La oferta de trabajo debía estar formulada de forma precisa y ajustada a los requerimientos del puesto de trabajo. Para ello, los ayuntamientos debían tomar como referencia las categorías profesionales y los criterios de realización asociados a alguna unidad de competencia incluida en el Catálogo Nacional de Cualificaciones Profesionales (CNCP) vigentes para definir la oferta de trabajo. Todo ello, con objeto de que la experiencia profesional adquirida en el desempeño del puesto de trabajo se

13 Estas son: grupo 1: 1.700 euros al mes; grupo 2: 1.600 euros al mes; grupo 3: 1.500 euros al mes; grupos 4-10: 1.300 euros al mes.

14 Adicionalmente, dentro de cada grupo de prioridad se ordenarán los candidatos atendiendo a la mayor disponibilidad para el empleo, la fecha de solicitud de ocupación o, en su defecto, a la fecha de inscripción, de la más antigua a la más reciente. 
pudiese acreditar a posteriori. En este sentido, esta iniciativa introdujo un aspecto novedoso respecto a sus convocatorias precedentes: el proceso de tutorización de las personas contratadas ${ }^{15}$. Es decir, a la finalización del período de contratación, el ayuntamiento debía elaborar y entregar un certificado individual a cada participante en el que quedase constancia de las competencias profesionales adquiridas. Finalmente, se debía realizar un informe de seguimiento global del Proyecto de Cooperación Social y Comunitaria en el que se reflejaran los resultados obtenidos. Este documento debía ser entregado al SAE junto con la justificación económica de la ayuda ${ }^{16}$.

En relación con el tipo de contrato que debía usar el ayuntamiento, el programa definió la modalidad de contrato de obra o servicio determinado. La duración del contrato dependía del programa. En el programa Emple@30+ se estableció un período mínimo de tres meses, debiendo formalizarse por meses completos, hasta un máximo de seis.En el programa Emple@Joven, la duración mínima establecida fue de seis meses. Además, los contratos se tenían que concertar a jornada completa.

En cuanto a los requisitos y criterios de las personas participantes, estas tenían que estar inscritas como demandantes de empleo no ocupadas y ser residentes en el municipio de referencia. En el programa Emple@30+ también se exigía que el participante estuviese inscrito en el fichero del Sistema Nacional de Garantía Juvenil.

\subsection{Perfil de los contratados (grupo de tratamiento)}

La base de datos que se usa para realizar la evaluación de impacto procede del SAE, tanto la relativa a los participantes del programa como al grupo de control que se describirá en la siguiente sección. Recordemos que, dado el funcionamiento del programa, el SAE tiene toda la información estadística de los demandantes de empleo que se han beneficiado de las iniciativas locales de empleo. El número global de personas contratadas por los ayuntamientos a julio de 2018 ascendió a un total de 33.295 personas, de las cuales 17.770 eran participantes en el programa empleo joven y 15.525 participantes en el programa $30+$.

Comenzamos presentando en el Cuadro 1 la composición de los beneficiarios de las iniciativas locales de empleo para cada uno de los programas según un conjunto de características socioeconómicas. Dichas características se concretan en el siguiente conjunto de variables observables: género (mujer), edad, niveles de estudio (tres niveles de estudio: educación primaria, secundaria y postsecundaria), nacionalidad (inmigrante frente a nativo), beneficiario del IMS (IMS, variable ficticia que toma valor la unidad si es beneficiario y cero en caso contrario), duración del paro

15 El proceso de tutorización consiste fundamentalmente en cumplimentar el cuaderno de seguimiento, donde se debe detallar las realizaciones profesionales incluidas en cualificaciones vigentes del CNCP al objeto de que se pueda realizar un seguimiento de las competencias adquiridas con la práctica laboral.

${ }_{16}$ A las obligaciones anteriores, habría que sumar obligaciones relativas a la contabilidad de los gastos, ejecución de la obra o servicio, y obligaciones de información y publicidad establecidas por la normativa comunitaria y el Servicio Andaluz de Empleo, entre otras que se mencionan en la convocatoria. 


\section{CUADRO 1 \\ CARACTERÍSTICAS SOCIOECONÓMICAS DE LOS PARTICIPANTES}

(En \%)

\begin{tabular}{|c|c|c|c|}
\hline & & $\begin{array}{c}\text { 30+ } \\
\text { Media (sd) }\end{array}$ & $\begin{array}{c}\text { Joven } \\
\text { Media (sd) }\end{array}$ \\
\hline & Mujer (\%) & $46(0,50)$ & $51(0,50)$ \\
\hline & Edad (años) & $44,5(7,9)$ & $24,1(3,4)$ \\
\hline & Educación primaria & $9(0,28)$ & $6(0,39)$ \\
\hline & Educación secundaria & $57(0,50)$ & $65(0,48)$ \\
\hline & Educación postsecundaria & $35(0,48)$ & $37(0,48)$ \\
\hline & Inmigrante & $2(0,13)$ & $1(0,10)$ \\
\hline & Beneficiario IMS & $22(0,41)$ & $7(0,26)$ \\
\hline & Minusválido & $4(0,18)$ & $4(0,20)$ \\
\hline & Duración del paro (meses) & $13,9(9,1)$ & $12,2(8,7)$ \\
\hline & $12-23$ meses & $20(0,40)$ & $25(0,43)$ \\
\hline & $>=24$ meses & $35(0,48)$ & $23(0,42)$ \\
\hline & Sin experiencia laboral & $11(0,31)$ & $35(0,48)$ \\
\hline & Meses experiencia laboral & $47,8(61,8)$ & $5,3(10,7)$ \\
\hline & Colectivo CDIL & $69(0,46)$ & $63(0,48)$ \\
\hline & Tratado L2014 & $11(0,31)$ & $19(0,39)$ \\
\hline \multirow{6}{*}{ Tamaño municipio } & $<5$ mil & $22(0,41)$ & $14(0,35)$ \\
\hline & 5-10 mil & $14(0,34)$ & $11(0,32)$ \\
\hline & $10-20$ mil & $18(0,38)$ & $16(0,37)$ \\
\hline & $20-50 \mathrm{mil}$ & $18(0,38)$ & $20(0,40)$ \\
\hline & $50-100 \mathrm{mil}$ & $11(0,32)$ & $14(0,34)$ \\
\hline & $>100$ mil & $18(0,38)$ & $24(0,43)$ \\
\hline \multirow{8}{*}{ Provincia } & Almería & $8(0,27)$ & $8(0,27)$ \\
\hline & Cádiz & $13(0,34)$ & $16(0,37)$ \\
\hline & Córdoba & $11(0,31)$ & $11(0,31)$ \\
\hline & Granada & $13(0,33)$ & $11(0,32)$ \\
\hline & Huelva & $7(0,27)$ & $7(0,25)$ \\
\hline & Jaén & $11(0,31)$ & $9(0,29)$ \\
\hline & Málaga & $14(0,34)$ & $14(0,35)$ \\
\hline & Sevilla & $23(0,42)$ & $23(0,42)$ \\
\hline \multicolumn{2}{|l|}{ Total } & 17.770 & 15.525 \\
\hline
\end{tabular}

FUENTE: Elaboración propia con los datos procedentes del SAE. 
(meses), experiencia laboral, beneficiario de una iniciativa local de empleo anterior (Tratado L2014), tamaño del municipio (seis variables ficticias de tamaño de municipio según número de habitantes) y provincia de residencia. También construimos una variable Colectivo CDIL, que toma valor la unidad si se trata de individuos que por sus características demográficas se pueden catalogar de vulnerables y, por tanto, con dificultades de inserción laboral, y cero en caso contrario. Dichos colectivos son PLD (duración del episodio de paro superior a 12 meses), individuos con estudios primarios, inmigrantes o perceptores del IMS ${ }^{17}$.

El Cuadro 1 muestra que el programa cumple uno de sus objetivos fundamenta$l_{\text {les }}{ }^{18}$, este es, dirigirse a Colectivos CDIL. Este colectivo representa más del $60 \%$ de los beneficiarios en ambos programas (el $69 \%$ en los mayores de 30 y del $63 \%$ en los jóvenes). Como era de esperar, entre los participantes hay un alto porcentaje de PLD (el $55 \%$ en el caso del programa de mayores de 30 años y el $48 \%$ en el caso del programa para jóvenes). En el programa empleo 30+ los beneficiarios del IMS representan el $22 \%$. En el programa empleo joven, ese porcentaje no es tan elevado porque para ser beneficiario del IMS se debe tener cargas familiares y hay muchos jóvenes que naturalmente no se encuentran en esa situación. Por tanto, respecto al potencial efecto de estos programas, la composición de la muestra de tratados tiene una lectura muy positiva.

\subsection{Descripción estadística del programa: características del programa}

En el Cuadro 2 se presenta una estadística básica del tipo de empleo realizado por el individuo beneficiario del programa. El tipo de empleo se caracteriza a partir de las siguientes variables: tipo de contrato (obra y servicio, eventual), duración del contrato, tipo de ocupación y año del contrato subvencionado. La duración del episodio de empleo se describe con cuatro tramos: entre 1 y 3 meses, entre 4 y 6 meses, entre 7 y 12 meses y más de 12 meses. El tipo de ocupación se agrupa en tres categorías principales: alta (grupos de cotización 1-3), media (grupos de cotización 4-7) y baja (grupos de cotización 8 y 9). Finalmente, para enmarcar temporalmente el análisis, los episodios de empleo hacen referencia al periodo que transcurre desde el comienzo del programa, este es noviembre de 2016, hasta agosto de 2018.

La duración observada de los contratos está íntimamente ligada a los periodos mínimos de contratación establecidos por la normativa reguladora para cada uno de los programas (6 meses para el programa joven y 3 meses para el 30+). Si analizamos el periodo de duración de las contrataciones con más detenimiento, se observa

\footnotetext{
17 Dentro de esta base de datos de individuos tratados proporcionada por el SAE tenemos algunas situaciones «anómalas» o poco comunes que se eliminarán de la base de datos objeto de análisis. En concreto, estas son: $i$ ) individuos con varios tratamientos (=varios contratos bajo el programa), nos quedamos con el último episodio; ii) minusválidos; iii) VVG; $i v$ ) individuos con contratos (tratamiento) de duración indeterminada.

${ }^{18}$ El programa define los colectivos prioritarios - es decir, en caso de tener que ordenar a los demandantes de empleo, los trabajadores con estas características tienen mayor probabilidad de beneficiarse del programa-, los cuales son el ser beneficiarios del IMS y ser PLD (> 12 meses).
} 


\section{CUADRO 2 \\ CARACTERÍSTICAS DEL EMPLEO}

(En \%)

\begin{tabular}{|c|c|c|c|}
\hline & & $\begin{array}{c}\text { 30+ } \\
\text { Media (sd) }\end{array}$ & $\begin{array}{c}\text { Joven } \\
\text { Media (sd) }\end{array}$ \\
\hline \multirow{4}{*}{ Duración del contrato } & $1-3$ meses & $42(0,5)$ & $4(0,2)$ \\
\hline & 4-6 meses & $53(0,5)$ & $87(0,3)$ \\
\hline & $7-12$ meses & $4(0,2)$ & $8(0,2)$ \\
\hline & $>12$ meses & $1(0,0)$ & $0(0,06)$ \\
\hline \multirow{3}{*}{$\begin{array}{l}\text { Tipo de ocupación } \\
\text { (cualificación) }\end{array}$} & Alta & $29(0,4)$ & $32(0,4)$ \\
\hline & Media & $43(0,5)$ & $31(0,4)$ \\
\hline & Baja & $28(0,4)$ & $37(0,5)$ \\
\hline \multirow{2}{*}{ Tipo de contrato } & Obra y servicio & $95(0,2)$ & $96(0,2)$ \\
\hline & Eventual y otros & $5(0,2)$ & $3(0,2)$ \\
\hline \multirow{3}{*}{$\begin{array}{l}\text { Año del contrato } \\
\text { subvencionado }\end{array}$} & 2016 & $2(0,1)$ & $2(0,1)$ \\
\hline & 2017 & $11(0,2)$ & $9(0,2)$ \\
\hline & 2018 & $87(0,3)$ & $89(0,3)$ \\
\hline \multirow{9}{*}{$\begin{array}{l}\text { Tipos de ocupación } \\
\text { (CNO-1d) }\end{array}$} & Directores y gerentes & 0,1 & 0,1 \\
\hline & Técnicos y profesionales & 19 & 17 \\
\hline & Técnicos, profesionales de apoyo & 9,9 & 15 \\
\hline & $\begin{array}{l}\text { Administrativos, empleos de } \\
\text { oficina }\end{array}$ & 5,3 & 7,9 \\
\hline & $\begin{array}{l}\text { Servicios de restauración, } \\
\text { vendedores }\end{array}$ & 6,2 & 5,8 \\
\hline & $\begin{array}{l}\text { Cualificado agrícola, ganadero, } \\
\text { forestal }\end{array}$ & 5,5 & 5,9 \\
\hline & $\begin{array}{l}\text { Artesanos/trabajadores } \\
\text { cualificados industria/construcción }\end{array}$ & 25 & 11 \\
\hline & $\begin{array}{l}\text { Operadores de instalaciones } \\
\text { y maquinaria }\end{array}$ & 6,2 & 0,1 \\
\hline & Ocupación elemental & 27 & 36 \\
\hline
\end{tabular}

FUENTE: Elaboración propia con los datos procedentes del SAE: extracción de la base de datos de demandantes de empleo del SAE.

que en el programa Emple@Joven predominó el periodo mínimo de contratación regulado por la norma (el $87 \%$ de las contrataciones han sido de 6 meses), mientras que en el Emple@30+ las contrataciones se extendieron más allá del periodo mínimo exigido de 3 meses (casi el $58 \%$ han sido contrataciones de más 3 meses, y de estas casi la mitad, el 46,8\%, han tenido una duración de 6 meses). Con relación a la jornada laboral, la mayoría de los contratos fueron a tiempo completo. Solo el 4,1\% de los contratos en el caso de los jóvenes y el 5,9\% en el programa $30+$ se realizaron a tiempo parcial. 
En las iniciativas locales de empleo, las ofertas de empleo generadas están fundamentalmente vinculadas a las competencias municipales. El carácter descentralizado de este tipo de programas permite que se generen una amplia variedad de tipos de empleo. Esta característica se pone de manifiesto con el análisis por gran grupo de ocupación ${ }^{19}$. La mayor proporción de las contrataciones se realizaron en ocupaciones elementales que requerían una baja especialización ${ }^{20}$ (un $27 \%$ en el programaEmple@30+y 36\% en el programaEmple@Joven). No obstante, hay dos categorías con una alta presencia que sí requiere una especialización, como es el caso de los artesanos y trabajadores cualificados de las industrias manufactureras y la construcción (25\% en el programa Emple@30+), y las ocupaciones de técnicos y profesionales científicos e intelectuales (19\% en el programa Emple@30+y 17\% en el programa Emple@Joven).

\subsection{Descripción estadística del programa: indicadores de resultados}

El principal indicador estadístico para medir la eficacia del programa en términos de mejora del nivel de empleabilidad es la probabilidad de salir del paro al empleo. Para evaluar dicha probabilidad, el SAE nos proporciona información sobre la situación laboral de los beneficiarios de las iniciativas locales de empleo seis meses después de haber finalizado el contrato. Es decir, la base de datos que nos proporciona el SAE nos permite identificar si el individuo está o no empleado seis meses después del contrato, así como algunas características del empleo.

Una posible objeción a esta forma de presentar la información es que es posible que un participante sea clasificado como no empleado tras el programa si ha tenido una experiencia de empleo corta que ha finalizado antes del momento de la recogida de la información de empleabilidad por parte del $\mathrm{SAE}^{21}$.

Para medir la eficacia del programa a medio plazo, tratamos también de evaluar la estabilidad del nuevo empleo y/o calidad del mismo. Con este fin se analizan algunas características del nuevo empleo tales como el tipo de contrato (indefinido frente a temporal), la jornada laboral (tiempo completo frente a tiempo parcial), tipo de cualificación en el empleo (medida por el grupo de tarifa) o duración del contrato. Con esta información se puede evaluar, por ejemplo, si el programa ha favorecido cierta estabilidad laboral (i.e. el individuo tratado ha conseguido un contrato indefinido tras su participación en el programa) o ha favorecido mejoras en la cualificación ${ }^{22}$.

${ }^{19}$ El tipo de ocupación se basa en la Clasificación Nacional de Ocupaciones a un dígito.

20 Entre los diferentes tipos de empleo, ocupa un lugar predominante la reparación y mantenimiento de calles, jardines y otros espacios/edificios públicos.

21 Dado que el objetivo del programa es medir la empleabilidad del individuo a medio plazo (más que a corto plazo), esta forma de recoger la información es apropiada y no debería introducir sesgos importantes en el análisis de los resultados.

22 Otro indicador interesante sería la duración del nuevo empleo, sobre todo si es de duración superior a un tiempo mínimo. 
En el Cuadro 3 se presentan los principales indicadores de eficiencia del programa. Estos son la probabilidad de salida del paro y el grado de estabilidad del nuevo empleo según los indicadores arriba mencionados. De los 33.295 beneficiarios del programa $30+$, un total de 9.801 han obtenido un contrato en los seis meses posteriores a la finalización del programa, lo que depara una tasa de contratación posterior al programa del 29,4\%. En el caso de los beneficiarios del programa empleo joven, los resultados son aún más favorables y el $33 \%$ de los participantes tienen un contrato seis meses después del programa. Dicho de otro modo, casi 1 de cada 3 participantes en ambos programas estaba empleado a los seis meses de finalización del mismo.

De los que consiguen un empleo, la mayoría lo hacen con un contrato temporal (97-98\% dependiendo del programa). Esto se corresponde con la dinámica propia del mercado de trabajo español, donde más del $95 \%$ de los nuevos contratos son temporales. En relación con la jornada laboral, un $47 \%$ tienen un empleo a tiempo parcial en el programa Emple@30+, mientras que un $60 \%$ tienen un empleo a tiempo parcial tras el programa Emple@Joven. Por tanto, la incidencia en cuanto a parcialidad es muy alta, sobre todo en el colectivo de los jóvenes ${ }^{23}$. La última medida de empleabilidad presentada tiene que ver con la eventual ocupación posterior en los seis meses posteriores a la finalización del programa. La mayoría de las ocupaciones están en los niveles bajos (48\% para el programa Emple@30+) o medios (43\% para el programa Emple@ Joven).

\section{CUADRO 3 \\ INDICADORES DE INSERCIÓN LABORAL TRAS EL PROGRAMA} (En \%)

\begin{tabular}{|l|l|c|c|}
\hline \multicolumn{2}{|l|}{} & \multicolumn{1}{|c|}{$\begin{array}{c}\text { 30+ } \\
\text { Media (sd) }\end{array}$} & $\begin{array}{c}\text { Joven } \\
\text { Media (sd) }\end{array}$ \\
\hline \multicolumn{2}{|l|}{ Sale del paro al empleo } & $29(0,45)$ & $33(0,47)$ \\
\hline \multirow{3}{*}{ Condicionado a salir del paro } & Contrato temporal & $98(0,14)$ & $97(0,18)$ \\
\cline { 2 - 4 } & Tiempo parcial & $47(0,50)$ & $60(0,49)$ \\
\hline \multirow{4}{*}{ Duración del contrato } & $<3$ meses & $34(0,46)$ & $34(0,45)$ \\
\cline { 2 - 4 } & $3-6$ meses & $6(0,28)$ & $9(0,28)$ \\
\cline { 2 - 4 } & Con fecha de finalización & $45(0,50)$ & $55(0,50)$ \\
\hline \multirow{3}{*}{ Tipo de ocupación } & Alta & $13(0,34)$ & $20(0,40)$ \\
\cline { 2 - 4 } & Media & $39(0,49)$ & $43(0,50)$ \\
\cline { 2 - 4 } & Baja & $48(0,50)$ & $37(0,48)$ \\
\hline
\end{tabular}

FUENTE: Elaboración propia con los datos procedentes del SAE: extracción de la base de datos de demandantes de empleo del SAE.

${ }^{23}$ Según la EPA, la tasa de empleo a tiempo parcial en Andalucía en esos años se sitúa en torno al 16,2\% (14,8\% para el caso de España). 


\section{Descripción de la metodología de la evaluación de impacto: efecto medio del tratamiento}

Si bien el uso de la estadística descriptiva presentada en la sección anterior puede ser indicativa de ciertas bondades de esta iniciativa, no es posible extraer conclusiones robustas sobre la efectividad del programa sobre los niveles de empleabilidad de los beneficiarios. Para ello, es necesario realizar un análisis contrafactual que permita testar la existencia de una relación causal entre la participación en el programa y la ganancia en empleabilidad posterior de los participantes. Es decir, hasta que no se demuestre que los resultados de inserción laboral de individuos iguales a los tratados, pero que no se han beneficiado del programa, son peores a los de los tratados. Esta es a la pregunta que se trata de contestar en el ejercicio de evaluación causal: ¿qué le hubiese pasado -en términos de duración total del episodio de paro, probabilidad de encontrar un empleo o probabilidad de engrosar las listas de inactivos- a los desempleados participantes de las iniciativas locales de empleo $\left(Y_{1 i}\right)$ si no hubieran participado en la misma? Esta última situación es lo que se denomina, resultado «contrafactual $\ll\left(Y_{0 i}\right)$.

Para poder valorar la diferencia entre el resultado observado $\left(Y_{1 i}\right)$ y el «contrafactual» $\left(Y_{0 i}\right)$, la aproximación más habitual consiste en comparar la situación de dos grupos de individuos, uno de los cuales se ha beneficiado de la política (conocido como grupo de tratamiento o simplemente tratados, $Y_{1 i}$ ) y el otro no (conocido como grupo de control o simplemente controles, $Y_{0 i}$ ). Es decir, el efecto del tratamiento en el individuo se calcularía como la diferencia entre los dos resultados $\left(Y_{1 i}-Y_{0 i}\right)$. La naturaleza de estos estudios es del tipo cuasiexperimental pues, en la práctica, los individuos no son asignados de manera aleatoria a los grupos de tratamiento y control, sino que existen unos criterios de elegibilidad que determinan dicha asignación. En el caso del programa que evaluamos, la selección de los participantes depende del carácter más o menos vulnerable del individuo sobre la base de las variables renta -a través de la percepción o no del IMS- y desempleo -a través de la pertenencia al colectivo de PLD.

En este trabajo, como metodología de evaluación de impacto, utilizamos la técnica conocida como método de emparejamiento basado en la probabilidad de asignación al tratamiento (propensity score matching, PSM ${ }^{24}$. El método de emparejamiento consiste en hacer parejas (matches) entre personas del grupo de tratamiento y personas del grupo de control con algunas características de partida similares, para después comparar sus resultados finales y, posteriormente, estimar el efecto medio

${ }^{24}$ Se utiliza esta técnica al no ser posible realizar otro tipo de análisis en condiciones experimentales que permitirían distribuir los «tratamientos» de forma aleatoria y estimar los efectos netos de manera más precisa. No obstante, este método permite simular esas condiciones mediante un diseño cuasiexperimental (o de experimento natural) para aproximarse más al efecto neto real de la intervención. Asimismo, se elige este procedimiento frente a otros análisis cuasiexperimentales por la falta de una regla clara y repentina de aplicación de la intervención (diseño de regresión discontinua) o de una evolución similar previa de dos grupos que luego puedan divergir a partir del tratamiento (diferencias-en-diferencias), entre otros aspectos. 
del tratamiento (average treatment effect). El aspecto clave del procedimiento de emparejamiento consiste en un algoritmo que permite identificar, con la máxima precisión posible, aquellos individuos no tratados parecidos a cada uno de los participantes en el programa. Este procedimiento de emparejamiento se basa en un modelo econométrico que estima la probabilidad de ser beneficiario del programa, a partir de la muestra de tratados y no tratados, y usando como variables explicativas de dicha probabilidad un conjunto de características observables.

A partir de dicho algoritmo se identifica una muestra de individuos contrafactuales, que en base a la probabilidad estimada de ser tratado - misma propensión a participar en el programa-, es casi igual al grupo de individuos tratados (Smith \& Todd, 2005). Al emparejar a los individuos tratados con contrafactuales con la «misma o parecida» probabilidad de participar en el programa, se evita el problema del sesgo de selección: es decir, el hecho de que las personas que participaron en el programa fueran diferentes en sus niveles de inserción laboral potencial frente al conjunto de los individuos sin trabajo. Una vez se ha hecho el «emparejamiento» de tratados y controles, se comparan los resultados del grupo «tratado» en este caso de participantes frente a los de los casos «de control» y se estima el efecto medio del tratamiento sobre los tratados (average treatment effect on the treated, ATET).

\section{Radiografía de los grupos de tratamiento y control}

Cuando hemos descrito el programa, hemos hecho una caracterización inicial de los participantes de las iniciativas locales del empleo. En esta sección presentamos un análisis comparativo entre la muestra del grupo de tratamiento y el grupo de control, con la finalidad de observar si el perfil de participantes en cada uno de los programasEmple@30+y Emple@Joven responde al patrón de desempleo que caracteriza a la región andaluza.

La estadística presentada en la sección 3 parece indicar que las iniciativas de empleo evaluadas están orientadas de forma efectiva a los colectivos más desfavorecidos, siendo estos los conformados por personas desempleadas con menor probabilidad de encontrar un trabajo en el medio plazo. Como se ha descrito en el punto anterior, la convocatoria de las iniciativas locales de empleo precisa unos criterios de selección que ordena a los potenciales beneficiarios de más a menos vulnerable. Por tanto, la participación en estos programas no es aleatoria y se basa en un conjunto de variables observables. Esto implica que una mera comparación de los resultados de los individuos beneficiarios y no beneficiarios del programa puede llevar a conclusiones erróneas dadas las especiales características del colectivo de beneficiarios.

La base de datos de partida para construir la muestra de individuos que componen el grupo de control la construye el SAE a partir de los demandantes de empleo registrados. Más concretamente, el SAE realiza una extracción aleatoria de los demandantes de empleo registrados a fecha de 30/06/2018, excluyendo a los participantes en otros programas de la Ley $2 / 2015$. El SAE establece como fecha de corte para la 
extracción de la muestra ese mes, al corresponderse con el mes de mayor volumen de participantes que finalizan el programa, en concreto el 49,8\%. Por tanto, para la contabilización del tiempo de búsqueda de empleo, se considera junio de 2018 como el momento 0 para el grupo de control y la propia finalización del programa para el grupo de tratamiento. En ambos casos, tanto para los beneficiarios del programa -tratado-, como para los no beneficiarios -controles- la información socioeconómica usada en este trabajo procede del SAE.

La dimensión de la muestra de individuos que forman parte del grupo de control que nos proporciona el SAE es adecuada (Cuadro 4).Para el programa Emple@30+ disponemos de 134.303 observaciones y para el programa Emple@Joven de 39.981. Esto significa que del total de jóvenes demandantes de empleo que componen la muestra que usamos en el ejercicio de impacto, el $70 \%$ pertenecen al grupo de control y, del total de mayores de 30 años, el $90 \%$ pertenecen al grupo de control. En consecuencia, para cada unidad de tratado habrá, con una alta probabilidad, un número suficiente de individuos en la muestra de control.

En el Cuadro 5 comparamos las características socioeconómicas de los beneficiarios del programa con las del grupo de no tratados. En dicho cuadro presentamos la media -si es una variable continua- o el porcentaje -si es una variable discreta-, para ambos grupos de individuos, así como la significatividad estadística del test de diferencias de medias. Si bien se observa una relativa homogeneidad entre ambos grupos, emergen ciertos aspectos diferenciados de interés, en parte consecuencia de los propios criterios de priorización establecidos por la convocatoria para la participación en el programa. Más concretamente, el análisis estadístico del Cuadro 6 confirma que el grupo de individuos participantes en cada uno de los programas es diferente de forma estadísticamente significativa a la de controles. Dichas diferencias apuntan que el programa se ha dirigido a colectivos con especiales dificultades de inserción laboral dentro del panorama del mercado laboral andaluz.

\section{CUADRO 4 \\ ESTADÍSTICA BÁSICA: COMPOSICIÓN DE LA MUESTRA DEL EJERCICIO DE EVALUACIÓN}

(En \%)

\begin{tabular}{|l|c|c|c|}
\hline Programa & Control & Tratado & Totales \\
\hline \multirow{2}{*}{$30+$} & 134.303 & 13.819 & 148.122 \\
\cline { 2 - 4 } & 90,6 & 9,13 & 100 \\
\hline \multirow{2}{*}{ Joven } & 39.981 & 16.480 & 56.461 \\
\cline { 2 - 4 } & 70,8 & 28 & 100 \\
\hline \multirow{2}{*}{ Total } & $\mathbf{1 7 4 . 2 8 4}$ & $\mathbf{2 8 . 7 7 1}$ & $\mathbf{2 0 4 . 5 8 3}$ \\
\cline { 2 - 4 } & $\mathbf{8 5 , 2}$ & $\mathbf{1 4 , 8}$ & $\mathbf{1 0 0}$ \\
\hline
\end{tabular}

FUENTE: Elaboración propia a partir de los datos proporcionados por el SAE: extracción de la base de datos de demandantes de empleo del SAE. 


\section{CUADRO 5}

\section{ESTADÍSTICA DESCRIPTIVA CARACTERÍSTICAS SOCIOECONÓMICAS: DIFERENCIAS ENTRE LOS BENEFICIARIOS Y NO BENEFICIARIOS DEL PROGRAMA}

(En \%)

\begin{tabular}{|c|c|c|c|c|c|c|}
\hline & \multicolumn{3}{|c|}{$30+$} & \multicolumn{3}{|c|}{ Joven } \\
\hline & $\begin{array}{c}\begin{array}{c}\text { Control Mean } \\
\text { (sd) }\end{array} \\
\end{array}$ & $\begin{array}{c}\text { Tratado Mean } \\
\text { (sd) }\end{array}$ & $\begin{array}{c}\text { Diff } \\
\text { t-stat }\end{array}$ & $\begin{array}{c}\begin{array}{c}\text { Control Mean } \\
\text { (sd) }\end{array} \\
\end{array}$ & \begin{tabular}{|c|} 
Tratado Mean \\
(sd)
\end{tabular} & $\begin{array}{c}\text { Diff } \\
\text { t-stat }\end{array}$ \\
\hline Mujer & $61(0,49)$ & $47(0,50)$ & $* * *$ & $56(0,50)$ & $51(0,50)$ & $* * *$ \\
\hline$<20$ años & - & & & $13(0,33)$ & $4(0,18)$ & $* * *$ \\
\hline 20-24 años & - & & & $34(47)$ & $35(0,48)$ & $* * *$ \\
\hline 25-29 años & - & & & $54(0,50)$ & $62(0,49)$ & $* * *$ \\
\hline 30-39 años & $30(0,46)$ & $30(0,46)$ & - & & & \\
\hline 40-49 años & $35(0,48)$ & $38(0,49)$ & $* * *$ & - & & \\
\hline 55-59 años & $17(0,37)$ & $12(0,32)$ & $* * *$ & - & & \\
\hline Educación primaria & $20(0,40)$ & $9(0,28)$ & $* * *$ & $19(0,39)$ & $6(0,24)$ & $* * *$ \\
\hline Educación secundaria & $66(0,48)$ & $57(0,5)$ & $* * *$ & $65(0,48)$ & $56(0,50)$ & $* * *$ \\
\hline Educación superior & $15(0,35)$ & $34(0,48)$ & $* * *$ & 16 & $37(0,48)$ & $* * *$ \\
\hline Inmigrante & $8(0,2)$ & $2(0,1)$ & $* * *$ & $7(0,2)$ & $1(0,1)$ & $* * *$ \\
\hline IMS & $1(0,1)$ & $22(0,4)$ & $* * *$ & $1(0,1)$ & $7(0,2)$ & $* * *$ \\
\hline Minusválido & $3(0,2)$ & $4(0,1)$ & $* * *$ & $3(0,1)$ & $4(0,2)$ & $* * *$ \\
\hline $\begin{array}{l}\text { Duración paro } \\
1-3 \text { meses }\end{array}$ & $24(0,4)$ & $16(0,3)$ & $* * *$ & $45(0,5)$ & $18(0,3)$ & $* * *$ \\
\hline 4-6 meses & $14(0,3)$ & $13(0,3)$ & $* * *$ & $20(0,4)$ & $16(0,3)$ & $* * *$ \\
\hline $7-11$ meses & $17(0,3)$ & $17(0,3)$ & $* * *$ & $17(0,3)$ & $19(0,3)$ & $* * *$ \\
\hline $12-23$ meses & $15(0,3)$ & $20(0,4)$ & $* * *$ & $10(0,3)$ & $25(0,4)$ & $* * *$ \\
\hline$>=24$ meses & $30(0,4)$ & $35(0,4)$ & $* * *$ & $8(0,2)$ & $23(0,4)$ & $* * *$ \\
\hline Sin exp. laboral & $27(0,4)$ & $11(0,3)$ & $* * *$ & $39(0,4)$ & $35(0,4)$ & $* * *$ \\
\hline Meses exp. laboral & $35,5(59,7)$ & $47,8(61,8)$ & $* * *$ & $4,1(10,7)$ & $5,4(10,5)$ & $* * *$ \\
\hline $\begin{array}{l}\text { Colectivo } \\
\text { desfavorable (CDIL) }\end{array}$ & $65(0,4)$ & $68(0,4)$ & $* * *$ & $57(0,5)$ & $63(0,4)$ & $* * *$ \\
\hline Tratado L2014 & $2(0,1)$ & $11(0,3)$ & $* * *$ & $4(0,1)$ & $19(0,3)$ & $* * *$ \\
\hline $\begin{array}{l}\text { Tamaño municipio } \\
<5 \text { mil }\end{array}$ & $8(0,2)$ & $21(0,4)$ & $* * *$ & $10(0,3)$ & $14(0,3)$ & $* * *$ \\
\hline $5-10 \mathrm{mil}$ & $8(0,2)$ & $14(0,3)$ & $* * *$ & $9(0,2)$ & $11(0,3)$ & $* * *$ \\
\hline $10-20 \mathrm{mil}$ & $12(0,3)$ & $18(0,4)$ & $* * *$ & $13(0,3)$ & $16(0,3)$ & $* * *$ \\
\hline $20-50 \mathrm{mil}$ & $18(0,3)$ & $18(0,4)$ & $* *$ & $19(0,3)$ & $20(0,4)$ & $* * *$ \\
\hline $50-100 \mathrm{mil}$ & $16(0,3)$ & $12(0,3)$ & $* * *$ & $15(0,3)$ & $14(0,3)$ & $* * *$ \\
\hline$>100 \mathrm{mil}$ & $38(0,4)$ & $18(0,3)$ & $* * *$ & $35(0,4)$ & $24(0,4)$ & $* * *$ \\
\hline $\begin{array}{c}\text { Provincia } \\
\text { Almería }\end{array}$ & $8(0,2)$ & $8(0,2)$ & & $8(0,2)$ & $8(0,2)$ & \\
\hline Cádiz & $18(0,3)$ & $14(0,3)$ & $* * *$ & $17(0,3)$ & $16(0,3)$ & $*$ \\
\hline Córdoba & $9(0,2)$ & $11(0,3)$ & $* * *$ & $10(0,3)$ & $11(0,3)$ & $* * *$ \\
\hline Granada & $10(0,3)$ & $13(0,3)$ & $* * *$ & $11(0,3)$ & $11(0,3)$ & \\
\hline Huelva & $7(0,2)$ & $8(0,2)$ & $* * *$ & $6(0,2)$ & $7(0,2)$ & $* * *$ \\
\hline Jaén & $6(0,2)$ & $10(0,3)$ & $* * *$ & $8(0,2)$ & $9(0,2)$ & $* * *$ \\
\hline Málaga & $18(0,3)$ & $14(0,3)$ & $* * *$ & $16(0,3)$ & $14(0,3)$ & $* * *$ \\
\hline Sevilla & $25(0,4)$ & $23(0,4)$ & $* * *$ & $24(0,4)$ & $23(0,4)$ & $* * *$ \\
\hline
\end{tabular}

FUENTE: Elaboración propia a partir de los datos proporcionados por el SAE: extracción de la base de datos de demandantes de empleo del SAE.

NOTA: *** Significativo al $1 \%$. ** Significativo al $5 \%$. $*$ Significativo al $10 \%$. 


\section{CUADRO 6}

ESTADÍSTICA DESCRIPTIVA DE LOS INDICADORES DE INSERCIÓN LABORAL TRAS EL PROGRAMA: DIFERENCIAS ENTRE LOS BENEFICIARIOS Y NO BENEFICIARIOS DEL PROGRAMA

(En \%)

\begin{tabular}{|l|c|c|c|c|c|c|c|}
\hline \multirow{2}{*}{} & \multicolumn{2}{|c|}{$30+$} & \multicolumn{3}{|c|}{ Joven } \\
\cline { 2 - 8 } & $\begin{array}{c}\text { Control } \\
\text { Mean (sd) }\end{array}$ & $\begin{array}{c}\text { Tratado } \\
\text { Mean (sd) }\end{array}$ & $\begin{array}{c}\text { Diff } \\
\text { t-stat }\end{array}$ & $\begin{array}{c}\text { Control } \\
\text { Mean (sd) }\end{array}$ & $\begin{array}{c}\text { Tratado } \\
\text { Mean (sd) }\end{array}$ & $\begin{array}{c}\text { Diff } \\
\text { t-stat }\end{array}$ \\
\hline Probabilidad estar empleado & $27(0,4)$ & $29(0,4)$ & $* * *$ & $42(0,5)$ & $33(47)$ & $* * *$ \\
\hline Tipo de contrato & $96(0,2)$ & $98(0,14)$ & $* * *$ & $96(0,20)$ & $97(0,18)$ & $* * *$ \\
\hline Contrato temporal & $56(0,5)$ & $47(0,5)$ & $* * *$ & $64(0,48)$ & $0,59(0,49)$ & $* * *$ \\
\hline Tiempo parcial & $36(0,4)$ & $34(0,4)$ & $* * *$ & $38(0,46)$ & $34(0,45)$ & $* * *$ \\
\hline Duración del contrato & $11(0,3)$ & $6(0,3)$ & $* * *$ & $12(0,39)$ & $9(0,35)$ & $* * *$ \\
\hline$<3$ meses & $54(0,5)$ & $45(0,5)$ & $* * *$ & $63(0,48)$ & $55(0,5)$ & $* * *$ \\
\hline 3-6 meses & $9(0,3)$ & $13(0,3)$ & $* * *$ & $12(0,33)$ & $20(0,4)$ & $* * *$ \\
\hline Con fecha finalización & $45(0,5)$ & $39(0,5)$ & $* * *$ & $47(0,50)$ & $43(0,5)$ & $* * *$ \\
\hline Tipo de ocupación & $46(0,5)$ & $48(0,5)$ & & $41(0,49)$ & $37(0,48)$ & $* * *$ \\
\hline Alta &
\end{tabular}

FUENTE: Elaboración propia a partir de los datos proporcionados por el SAE: extracción de la base de datos de demandantes de empleo del SAE.

NOTA: *** Significativo al $1 \%$. ** Significativo al $5 \%$. $*$ Significativo al $10 \%$.

Así, encontramos que los colectivos con alguna o varias dificultades de inserción laboral representan el $68 \%$ de los beneficiarios, 3 puntos porcentuales (pp, en adelante) por encima del peso relativo de estos colectivos en el grupo de control del programa Emple@30+. Las diferencias son aún mayores en el programa Emple@ Joven, al alcanzar este diferencial 5 pp (63\% para los tratados y $57 \%$ para los controles). En el colectivo de beneficiarios hay un alto porcentaje de PLD (55\% y $48 \%$ en la muestra 30+ y joven, respectivamente) y de beneficiarios del IMS (22\% y $7 \%$ en la muestra 30+ y joven, respectivamente), en comparación con la muestra de no beneficiarios (los PLD representan el $45 \%$ y $18 \%$ en la muestra $30+$ y en joven, respectivamente, y los beneficiarios del IMS representan el $1 \%$ en ambos casos). Este dato tiene una lectura positiva respecto al efecto que está teniendo dicha política en cuanto a la integración laboral de los colectivos más desfavorecidos. Estos son precisamente los que suelen presentar más dificultades de acceso al mercado de trabajo y, por tanto, los expuestos a una mayor vulnerabilidad y riesgo de exclusión social. 
Sí es cierto que en algunas otras dimensiones, tales como el género o el nivel educativo de los participantes, la composición de la muestra de control es algo diferente a la que se esperaría. Así, en el grupo de tratamiento hay menos mujeres y menos individuos con estudios primarios que en el grupo de control. Se esperaría que en el grupo de tratamiento hubiese más individuos con estudios primarios, desde el punto de vista de que estos pueden ser más vulnerables a la hora de acceder al mercado de trabajo.

\section{Resultados de la estimación: evaluación de impacto de las iniciativas locales de empleo}

El objetivo del trabajo de evaluación es analizar la eficacia de las iniciativas locales de empleo. A través de un ejercicio de evaluación de impacto, pretendemos determinar si el programa ha supuesto una mejora en los niveles de empleabilidad de los beneficiarios en el corto y en el medio plazo. Para realizar dicho ejercicio de evaluación usamos las técnicas econométricas de modelos de emparejamiento ( propensity score matching, PSM) descrito previamente ${ }^{25}$.

\subsection{Función de emparejamiento}

La técnica de evaluación que usamos es un algoritmo conformado por diferentes pasos. En la primera etapa estimamos la probabilidad de ser beneficiario del programa, a partir de la muestra de observaciones compuesta por los individuos beneficiarios del programa -tratados-, y los demandantes de empleo no beneficiarios del programa -controles-. En esta fase, el aspecto más delicado es seleccionar adecuadamente las variables explicativas que nos ayudan a predecir dicha probabilidad. Estas variables deben estar correlacionadas con la probabilidad de ser tratado, pero no pueden ser predictores perfectos de la asignación al tratamiento. Si ponemos este requisito en el contexto del tipo de programa que estamos evaluando, esto implica que es fundamental que las variables seleccionadas identifiquen correctamente «la dificultad de inserción laboral». Otro requisito importante es que las variables que formen parte de la función de emparejamiento no estén afectadas por el programa.

Siguiendo estas pautas básicas, así como usando la información relativa a los criterios de selección del programa, las variables explicativas -descritas la mayoría en el apartado de sección estadística- que forman parte del modelo probabilístico que define la función de emparejamiento son: edad, mujer, nivel educativo (estudios primarios, estudios secundarios, estudios superiores), inmigrante, experiencia laboral

${ }^{25}$ Para ese fin se hace uso del comando psmatch2 disponible en el software econométrico STATA (Leuven \& Sianesi, 2003). 
previa (meses), duración del paro (1-3 meses, 4-6 meses, 6-12 meses, 12-23 meses, $>24$ meses), beneficiario IMS, tamaño del municipio de residencia y provincia de residencia. La edad del individuo se modeliza a partir de un conjunto de variables ficticias que definen diferentes grupos de edad (<20, 20-25, 25-29, 30-39, 40-49, 50-59). La experiencia laboral previa también se discretiza en las siguientes categorías: sin experiencia laboral, entre 1-12 meses, 13-36 meses, 36-60 meses, > 60 meses. El tamaño de municipio de residencia se mide a partir del número de habitantes y recoge las diferentes categorías: <5, 5-20, 20-50, 50-100, >100 mil habitantes.

Para estimar la función de emparejamiento definimos una variable ficticia que toma valor la unidad si el individuo participa en el programa $(D=1)$, y cero si el individuo no participa en el programa $(\mathrm{D}=0)$. La función de emparejamiento se estima con un modelo de elección discreta, suponiendo que los errores siguen una distribución valor extremo tipo I, usando como variables explicativas las arriba indicadas. Una vez estimado el modelo, se computan las probabilidades de ser beneficiario del programa para cada individuo, tratado y control. Estas probabilidades se usan para encontrar un grupo de individuos no beneficiarios del programa que sirvan como contrafactual de cada uno de los individuos que sí se han beneficiado del programa. En la construcción de la muestra de contrafactuales usamos el criterio del soporte común. Esta condición garantiza que las observaciones del grupo de tratamiento tengan siempre observaciones de comparación «cercanas» en base a las características socioeconómicas usadas para definir la función de emparejamiento.

Los resultados para construir la función de emparejamiento se presentan en el Cuadro 7. La estimación del modelo econométrico relativo a la probabilidad de asignación al tratamiento permite identificar los factores que más peso tienen en la probabilidad de ser tratado. Estos factores son -en cuanto que influyen positivamente en dicha probabilidad de asignación- ser parado de larga duración, ser perceptor del IMS y residir en municipios pequeños frente a grandes. La estimación de este modelo nos permite también evaluar la calidad del emparejamiento y concluir que la calidad es buena ${ }^{26}$.

Tras el cálculo de la probabilidad de tratamiento (propensity score), se realiza el emparejamiento entre las observaciones del grupo de tratamiento y el grupo de control. La ventaja de la amplia muestra de controles se manifiesta en el alto porcentaje de observaciones dentro del soporte común. En el programa Emple@30+,el soporte común es del $95 \%$ (12 tratados y 5.670 controles fuera del soporte común), mientras que en el de Emple@Joven, el soporte común alcanza el 99\% (1 tratado y

${ }^{26}$ El ajuste de los modelos de regresión es bueno (pseudo-R2 Nagelkerke en torno a 0,25). Para el programa 30+, el porcentaje de casos correctos pronosticados como de «tratamiento» se sitúa en el $66 \%$ y en el $90 \%$ para el grupo de control. El porcentaje de «falsos positivos» es del $33 \%$ : casos de «control» pronosticados como de tratamiento. Para el programa joven, el porcentaje de casos correctos pronosticados como de «tratamiento» se sitúa en el $61 \%$ y para el grupo de control se sitúa en el $77 \%$. El porcentaje de «falsos positivos» es del $38 \%$ : casos de «control» pronosticados como de tratamiento. 


\section{CUADRO 7}

RESULTADOS DE LA ESTIMACIÓN DE LA FUNCIÓN DE EMPAREJAMIENTO POR TIPO DE PROGRAMA

(Modelo Logit: $Y=1$ si el individuo es tratado; $\mathbf{Y}=0$ si el individuo no es tratado)

\begin{tabular}{|c|c|c|}
\hline & 30+ & Joven \\
\hline Inmigrante & $-0,0647 * * *$ & $-0,176^{* * * *}$ \\
\hline Beneficiario IMS & $0,238 * * *$ & $0,379 * * *$ \\
\hline \multicolumn{3}{|l|}{ Duración del paro } \\
\hline 3-6 meses & $0,0210 * * *$ & $0,114 * * *$ \\
\hline 6-12 meses & $0,0135 * * *$ & $0,128 * * *$ \\
\hline $12-23$ meses & $0,0775 * * *$ & $0,271 * * *$ \\
\hline$>24$ meses & $0,107 * * *$ & $0,331 * * *$ \\
\hline \multicolumn{3}{|l|}{ Experiencia laboral } \\
\hline Sin experiencia & $-0,0722 * * *$ & $-0,0326 * * *$ \\
\hline $1-6$ meses & $-0,00924 * * *$ & $0,0983^{* * *}$ \\
\hline 6-12 meses & 0,00209 & $0,0837 * * *$ \\
\hline Minusválido & $0,00898 * *$ & $0,0465 * * *$ \\
\hline \multicolumn{3}{|l|}{ Edad } \\
\hline $40-49$ & $0,00652 * * *$ & \\
\hline $50-55$ & $0,0176^{* * *}$ & \\
\hline$>55$ & $-0,0127 * * *$ & \\
\hline$>20$ & & $0,127 * * *$ \\
\hline $20-24$ & & $0,117 * * *$ \\
\hline \multicolumn{3}{|l|}{ Educación } \\
\hline Primaria & $-0,0560 * * *$ & $-0,137 * * *$ \\
\hline Secundaria & $0,0832 * * *$ & $0,203 * * *$ \\
\hline \multicolumn{3}{|l|}{ Mujer } \\
\hline Mujer & $-0,0580 * * *$ & $-0,0873 * * *$ \\
\hline \multicolumn{3}{|l|}{ Mujer* Educación } \\
\hline Primaria & 0,000830 & $0,0272 * *$ \\
\hline Secundaria & $0,0394 * * *$ & $0,0295 * * *$ \\
\hline \multicolumn{3}{|l|}{ Educación $*$ Paro } \\
\hline Primaria paro 12-24 meses & $0,0509 * * *$ & $0,0733 * * *$ \\
\hline Primaria paro $>24$ meses & 0,00932 & $0,0766 * * *$ \\
\hline Secundaria paro $12-24$ meses & $0,0176 * *$ & $0,0573 * * *$ \\
\hline Secundaria paro $>24$ meses & $0,0252 * * *$ & $0,0417 * * *$ \\
\hline \multicolumn{3}{|l|}{ Mujer * Paro } \\
\hline Mujer paro 12-24 meses & $-0,0168 * * *$ & $0,0572 * * *$ \\
\hline Mujer paro $>24$ meses & $-0,00259$ & $0,0551 * * *$ \\
\hline \multicolumn{3}{|l|}{ Tamaño municipio } \\
\hline$<5$ miles & 0,000830 & $0,0272 * *$ \\
\hline 5-20 miles & $0,0394 * * *$ & $0,0295 * * *$ \\
\hline 20-50 miles & $0,0509 * * *$ & $0,0733 * * *$ \\
\hline 50-100 miles & 0,00932 & $0,0766 * * *$ \\
\hline
\end{tabular}

FUENTE: Elaboración propia a partir de los datos proporcionados por el SAE. NOTA: *** Significativo al $1 \%$. ** Significativo al $5 \%$. $*$ Significativo al $10 \%$. 
129 controles fuera del soporte común). Esta ventaja también se manifiesta en que el número medio de emparejamiento para cada participante es alto (38), siendo el número mínimo de 3 en el caso del programa Emple@Joven y de 8 en el programa Emple@30+. Los emparejamientos se han realizado con al menos 20 unidades de comparación y solo con aquellas observaciones que estén en un radio de 0,01 , para que dichos emparejamientos sean lo más semejantes posibles.

Con el objeto de asegurar la calidad adecuada de la función de emparejamiento, se ha verificado la condición de balanceo (balancing tests) en las características observadas entre la muestra de tratados y sus contrafactuales correspondientes. Es decir, que no hay diferencias estadísticamente significativas en la media de cada uno de los regresores entre los tratados y sus contrafactuales, una vez realizado el emparejamiento. Las Figuras 1 y 2 muestran que ambas propiedades se cumplen.

\section{FIGURA 1}

EVALUACIÓN DE LA CALIDAD DEL EMPAREJAMIENTO:

DISTRIBUCIÓN DE LA PROBABILIDAD DE RECIBIR EL TRATAMIENTO

PARA EL GRUPO DE TRATAMIENTO Y EL GRUPO DE CONTROL POR TIPO DE PROGRAMA
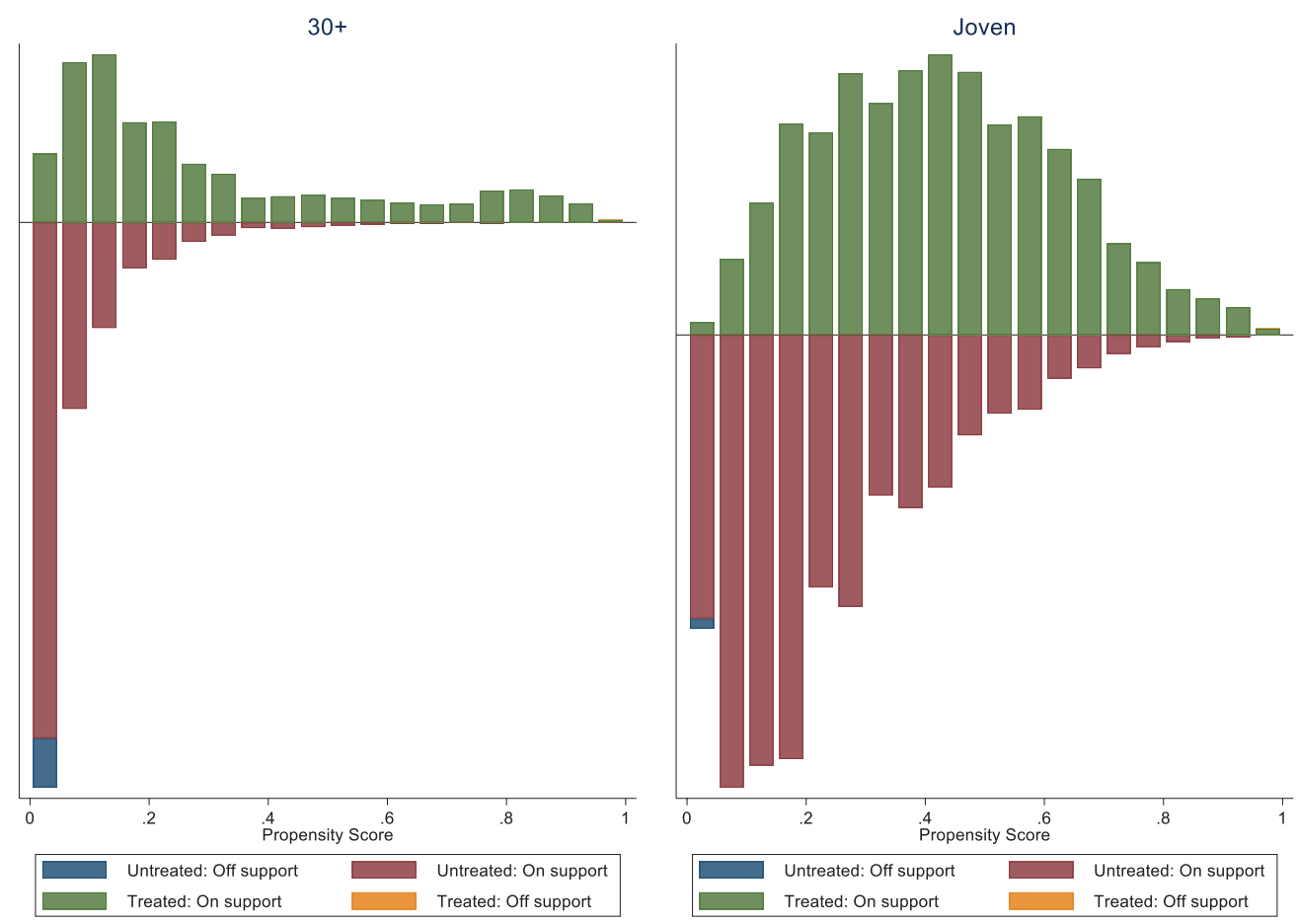

FUENTE: Elaboración propia a partir de los datos proporcionados por el SAE. 


\section{FIGURA 2}

\section{EVALUACIÓN DE LA CALIDAD DEL EMPAREJAMIENTO}
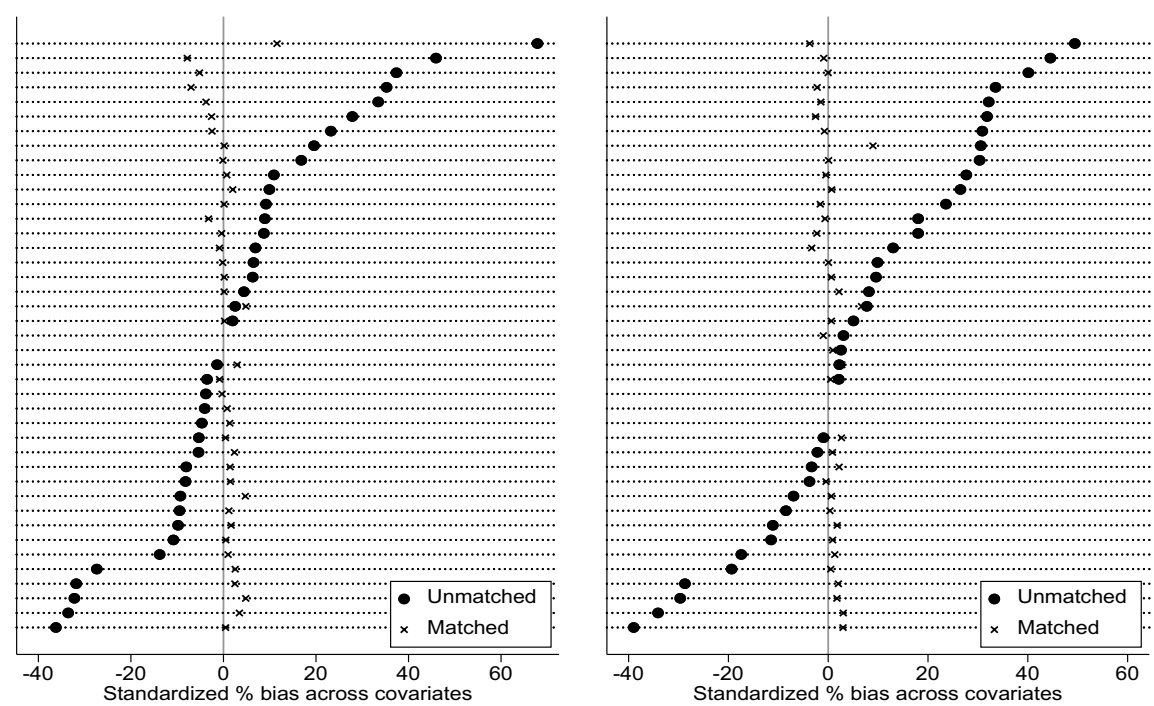

FUENTE: Elaboración propia a partir de los datos proporcionados por el SAE.

\subsection{Resultados de la evaluación: efecto medio del tratamiento}

Con los grupos de tratados y sus correspondientes contrafactuales, se realiza la evaluación de impacto del programa. Es decir, se comparan los resultados de las variables de interés entre el grupo de tratamiento y los contrafactuales seleccionados. Se seleccionan dos tipos de indicadores de resultados: la empleabilidad de los individuos y la calidad del nuevo empleo. La empleabilidad se mide a partir de la probabilidad de estar empleado seis meses después de ser beneficiario del programa de empleo (horizonte temporal de medio plazo). La calidad del nuevo empleo se mide a partir del tipo de contrato, la duración del contrato y el tipo de ocupación. El tipo de contrato se define en función de que se trate de un contrato temporal o indefinido. La idea es que, en media, un contrato indefinido garantiza una mayor estabilidad laboral que un contrato temporal. El tipo de ocupación se mide a partir de los grupos de cotización del nuevo contrato. Y finalmente, la duración del contrato se define como una variable binaria en base a una duración de menos o más de seis meses (corta duración frente a media/larga duración).

En el Cuadro 8 se presentan los resultados principales del ejercicio de evaluación para la muestra total de individuos y para la submuestra de individuos vulnerables. Más concretamente, se presenta la probabilidad -estimada- de estar empleado seis meses después de haber finalizado el contrato subvencionado para los tratados y sus contrafactuales, el efecto medio del tratamiento sobre los tratados estimado (ATT) 


\section{CUADRO 8}

\section{EFECTO CAUSAL DEL PROGRAMA SOBRE LA PROBABILIDAD DE OBSERVAR AL INDIVIDUO EMPLEADO SEIS MESES DESPUÉS DEL TRATAMIENTO (SALIDA DEL PARO AL EMPLEO)}

\begin{tabular}{|l|l|c|c|c|c|}
\hline \multicolumn{2}{|c|}{} & \multicolumn{2}{c|}{$\begin{array}{c}\text { Probabilidad de salir del } \\
\text { paro al empleo }\end{array}$} & \multicolumn{2}{c|}{ Diff } \\
\hline \multicolumn{2}{|c|}{ PSM } & Tratado & Control & ATT & T-stat \\
\hline $30+$ & Todos & 0,281 & 0,293 & $-0,012 * * *$ & $-2,3$ \\
\hline & CIDL & 0,211 & 0,199 & $0,012 * * *$ & 1,9 \\
\hline Joven & Todos & 0,325 & 0,378 & $-0,052 * * *$ & $-8,5$ \\
\hline & CIDL & 0,251 & 0,263 & $-0,012 * *$ & $-1,6$ \\
\hline
\end{tabular}

FUENTE: Elaboración propia a partir de los datos proporcionados por el SAE.

NOTA: *** Significativo al $1 \%$. ** Significativo al $5 \%$. * Significativo al $10 \%$. Boostrap standard errors.

y la significatividad estadística de dicho estimador. Estas estimaciones se presentan para cada programa (30+ y joven).

Lo primero que destaca en estos resultados es que el estimador ATT no parece ser positivo, es decir, no parece que el programa, en media, haya favorecido la salida del paro al empleo para los beneficiarios del programa. Así, para los jóvenes, los efectos estimados son de $-5,2$ pp para la muestra total y $-1,2$ pp para la submuestra de individuos con dificultades de inserción laboral. Para la muestra 30+, la probabilidad estimada de estar empleado tras el tratamiento es del 28,1\% para los tratados y, según el modelo, hubiese sido de un $29,3 \%$ para esa muestra de tratados en ausencia del programa. Esto es, el efecto causal estimado es que se reduce la probabilidad de estar empleado en 1,2 pp. Por el contrario, cuando nos centramos en la submuestra de individuos mayores de 30 con dificultades de inserción laboral, la probabilidad de estar empleado es del $21,1 \%$ y hubiese sido del 19,9\% en ausencia del tratamiento. Por tanto, es solo en este caso, parados mayores de 30 años con dificultades de inserción laboral, que encontramos un efecto causal positivo y significativo de en torno a $1,2 \mathrm{pp}$.

Los resultados indican, por tanto, que la política no parece estar beneficiando la empleabilidad de sus beneficiarios salvo para colectivos específicos. Los resultados presentados están en línea con la mayoría de la literatura empírica resumida a partir de los metaanálisis de Card, Kluve y Weber $(2010,2018)$ y Kluve (2010). En ellos se concluye que, en media, las políticas activas consistentes en la creación de empleo público no suelen llevar beneficios asociados a mayores niveles de empleabilidad de sus participantes. El análisis presentado no permite ofrecer una conclusión clara sobre los motivos o mecanismos que expliquen la falta de eficacia de esta política. La literatura empírica hace referencia a dos tipos de mecanismos, uno básicamente por el lado de la oferta de trabajo y otro por el lado de la demanda. En el primer caso, 
la literatura habla de los efectos de bloqueo (lock in effects). Esto es, los individuos quedan atrapados en este tipo de programas cuando la duración del programa es larga. Por otro lado, también se habla de los efectos estigma (stigma effects) que pueden sufrir los participantes de este tipo de políticas (Caliendo, 2006).

No obstante, es importante profundizar en el análisis, dado que dentro del colectivo de beneficiarios hay individuos con diferentes características y niveles potenciales de exclusión social, y esto puede dar lugar a una gran heterogeneidad de efectos causales. Por ello, en el Cuadro 9 se presentan los efectos medios -causales- del programa para diferentes tipos de individuos según género, nivel de estudios, edad, duración del episodio de paro, nacionalidad, beneficiario del IMS, por experiencia laboral y beneficiario del anterior programa de iniciativa de empleo local. En general, son todas ellas características socioeconómicas que nos ayudan a identificar mejor las situaciones de vulnerabilidad social y laboral.

Los resultados presentados en el Cuadro 9 apuntan la existencia de importantes diferencias en la incidencia del programa sobre la empleabilidad de los individuos beneficiarios del mismo. Comenzando con la duración del paro, vemos que los efectos positivos del programa aparecen para los individuos mayores de 30 años con episodios de paro de más de dos años. Para este colectivo, el programa ha aumentado la empleabilidad a medio plazo en 2,1 pp. Para otros colectivos, tales como los individuos mayores de 30 años sin experiencia laboral, los resultados también parecen ser positivos al aumentarles su empleabilidad en 3,1 pp. Por el contrario, para el resto de los colectivos y, en general, para los menores de 30 años, el programa no parece haber favorecido los niveles de empleabilidad de los beneficiarios.

Los resultados del Cuadro 9 también nos permiten indicar algunos factores que pueden ayudarnos a entender parte de los resultados encontrados. En concreto, es interesante destacar los impactos fuertemente negativos encontrados para los beneficiarios del IMS o para los individuos que también se habían beneficiado de iniciativas locales de empleo de convocatorias anteriores (programa en 2014, Tratado14=1). Ambos resultados apuntan a la existencia de que una parte de los beneficiarios se encuentran fuertemente vinculados a estos programas de ayuda social -lock in effects-, lo que puede desfavorecer una búsqueda activa de empleo y una menor tasa de llegada de ofertas.

Una vez presentados los resultados relativos a los efectos del programa sobre la empleabilidad de los individuos beneficiarios del mismo, pasamos a evaluar sus efectos a medio plazo y largo plazo. Más concretamente, se evalúa si el programa ha podido ayudar a mejorar la calidad del nuevo empleo (frente al empleo que hubiesen conseguido en ausencia del programa). Como se indicó anteriormente, las variables que usamos para medir la calidad del nuevo empleo son: tipo y duración del nuevo contrato, así como la cualificación del empleo.

El ejercicio de evaluación sigue los mismos pasos que los seguidos para evaluar la probabilidad de salir del paro. Los principales resultados se presentan en el Cuadro 10. La principal idea que emerge de dicho cuadro es que, en media, las características del empleo de los tratados pueden ser algo mejores que la de los controles. 


\section{CUADRO 9}

EFECTO CAUSAL DEL PROGRAMA SOBRE LA PROBABILIDAD DE OBSERVAR AL INDIVIDUO EMPLEADO SEIS MESES DESPUÉS DEL TRATAMIENTO POR CARACTERÍSTICAS SOCIOECONÓMICAS

\begin{tabular}{|c|c|c|c|}
\hline & & 30+ & Joven \\
\hline & & ATT & ATT \\
\hline \multirow{2}{*}{ Género } & Mujer & $-0,003$ & $-0,062 * * *$ \\
\hline & Hombre & $-0,020 * * *$ & $-0,027 * * *$ \\
\hline \multirow{3}{*}{ Formación } & Estudios primarios & $-0,002$ & $-0,054 * *$ \\
\hline & Estudios secundarios & $-0,008$ & $-0,047 * * *$ \\
\hline & Estudios superiores & $-0,023 * * *$ & $-0,035 * * *$ \\
\hline \multirow{6}{*}{ Edad } & $<20$ años & - & $-0,003$ \\
\hline & 20-24 años & - & $-0,040 * * *$ \\
\hline & 25-29 años & - & $-0,057 * * *$ \\
\hline & 30-39 años & 0,002 & - \\
\hline & 40-49 años & $-0,004$ & - \\
\hline & 50-59 años & $-0,033 * *$ & - \\
\hline \multicolumn{4}{|c|}{ Submuestra CDIL } \\
\hline \multirow{4}{*}{ Duración del paro } & $<6$ meses & $-0,057 * * *$ & $-0,086 * * *$ \\
\hline & 6-12 meses & $-0,045 * * *$ & $-0,059 * * *$ \\
\hline & 12- 24 meses & $-0,007$ & $-0,024 * * *$ \\
\hline & $>24$ meses & $0,021 * * *$ & $-0,010$ \\
\hline Inmigrante & & 0,006 & $-0,016$ \\
\hline Nativo & & $-0,015^{* * *}$ & $-0,045 * * *$ \\
\hline \multirow{2}{*}{ Beneficiado IMS } & Sí & $-0,028 * * *$ & $-0,096 * * *$ \\
\hline & No & $-0,008 * * *$ & $-0,047 * * *$ \\
\hline Minusválido & & 0,010 & $-0,006$ \\
\hline No minusválido & & $-0,014 * * *$ & $-0,047 * * *$ \\
\hline \multirow{2}{*}{ Experiencia laboral } & Sin experiencia. & $0,031 * * *$ & 0,008 \\
\hline & Con experiencia & $-0,021 * * *$ & $-0,079 * * *$ \\
\hline \multirow{2}{*}{ Programa 2014} & Tratado $14=1$ & $-0,086^{* * *}$ & $-0,096 * * *$ \\
\hline & Tratado $14=0$ & $-0,010 * *$ & $-0,035^{* * *}$ \\
\hline
\end{tabular}

FUENTE: Elaboración propia a partir de los datos proporcionados por el SAE.

NOTA: *** Significativo al $1 \%$. ** Significativo al $5 \%$. * Significativo al $10 \%$. Boostrap standard errors. 
Así, el Cuadro 10 muestra que el programa favorece la empleabilidad en empleos con contratos a tiempo completo y reduce la probabilidad de que dichos contratos sean de corta duración y/o de baja cualificación. Estos resultados podrían estar indicando que, para los colectivos donde el programa parece tener un impacto positivo en la empleabilidad, la inserción en el mercado de trabajo podría estar resultando a su vez en empleos de mayor estabilidad y duración. Sin embargo, hay que tomar este resultado con precaución dado que hemos visto como son pocos los colectivos que se benefician de estas medidas en términos de mayor empleabilidad, cuando lo comparamos con individuos comparables y no beneficiarios de la política.

En conclusión, por tanto, no se observa que, en media, el programa haya causado un aumento en la probabilidad de salir del paro al empleo seis meses después de haber participado en el programa. La heterogeneidad de los resultados es importante para el colectivo de mayores de 30 años, sobre todo, ya que hay determinados colectivos para los que los efectos sí parecen haber sido favorables, como son los parados de muy larga duración y las mujeres con estudios primarios/secundarios.

Por último, es importante tener en cuenta que, con el presente ejercicio de evaluación, no se evalúa si el programa ha evitado que los beneficiarios abandonen el mercado de trabajo, es decir, los efectos sobre la tasa de actividad. Dado que parte de los colectivos beneficiarios son individuos con altas dificultades de inserción laboral, un indicador complementario de esta evaluación sería evaluar sus efectos sobre la probabilidad de abandonar el mercado de trabajo. La falta de información estadística para medir esta variable de resultado impide arrojar luz a este respecto.

\section{CUADRO 10}

\section{EFECTO CAUSAL SOBRE OTROS INDICADORES DE EMPLEABILIDAD, SUBMUESTRA DE REEMPLEADOS}

\begin{tabular}{|c|c|c|c|c|c|c|c|}
\hline & & \multicolumn{2}{|c|}{$\begin{array}{l}\text { Todos } \\
\text { ATT }\end{array}$} & \multicolumn{2}{|c|}{$\begin{array}{l}\text { CDIL } \\
\text { ATT }\end{array}$} & \multicolumn{2}{|c|}{$\begin{array}{c}\text { Paro }>24 \text { meses } \\
\text { ATT }\end{array}$} \\
\hline & & $30+$ & Joven & $30+$ & Joven & $30+$ & Joven \\
\hline \multicolumn{2}{|c|}{ Tiempo completo } & $0,072 * * *$ & $0,058 * * *$ & $0,052 * * *$ & $0,051 * * *$ & $0,034 * * *$ & 0,002 \\
\hline \multirow{3}{*}{$\begin{array}{l}\text { Duración } \\
\text { del } \\
\text { contrato }\end{array}$} & $<3$ meses & $-0,071 * * *$ & $-0,065 * * *$ & $-0,059 * * *$ & $-0,076 * * *$ & $-0,057 * * *$ & $-0,079 * * *$ \\
\hline & 3-6 meses & $-0,040 * * *$ & $-0,016^{* * *}$ & $-0,014 * * *$ & $-0,014 * * *$ & $-0,031 * * *$ & $-0,020$ \\
\hline & Fecha final & $-0,138 * * *$ & $-0,090 * * *$ & $-0,163 * * *$ & $-0,096 * * *$ & $-0,143 * * *$ & $-0,082 * * *$ \\
\hline \multirow{3}{*}{$\begin{array}{l}\text { Cualif. del } \\
\text { empleo }\end{array}$} & Alta & 0,008 & 0,004 & $0,022 * * *$ & $0,016^{*}$ & 0,006 & $0,048 * * *$ \\
\hline & Media & $-0,027 * * *$ & $-0,015 * *$ & $0,051 * * *$ & 0,010 & $0,076^{* * *}$ & $-0,023$ \\
\hline & Baja & $0,019 * *$ & 0,011 & $-0,072 * * *$ & $-0,026^{* *}$ & $-0,082 * * *$ & $-0,026$ \\
\hline
\end{tabular}

FUENTE: Elaboración propia a partir de los datos proporcionados por el SAE.

NOTA: *** Significativo al $1 \%$. ** Significativo al $5 \%$. * Significativo al $10 \%$. Boostrap standard errors. 


\section{Conclusiones}

La evaluación de las PAE viene justificada por la necesidad de determinar la efectividad y la eficacia de estos programas. La efectividad en el logro de los objetivos propuestos y la eficacia en términos de relación entre el logro de objetivos y recursos invertidos para alcanzarlos. Además, solo a través de un análisis detallado de los programas se determinará sobre qué poblaciones tendrán una mayor incidencia y cuáles van a ser los mejores procesos para llevarlos a cabo, para su correcta puesta en marcha y ejecución.

La revisión de la literatura ha puesto de manifiesto que las iniciativas locales de empleo no funcionan en todas las circunstancias y con todos los desempleados. El diseño, la cobertura y los objetivos de estos programas, y el modo en que se aplican, pueden afectar mucho en términos de eficacia y eficiencia. No existe una única política que sirva como herramienta universal para mejorar las perspectivas laborales de los desempleados.

Las iniciativas locales de empleo analizadas en este trabajo se han diseñado de cara a lograr un alto grado de cobertura, tanto por la participación de las entidades beneficiarias (ayuntamientos andaluces) como por la participación de personas desempleadas no ocupadas inscritas como demandantes de empleo. Una gran parte de estos últimos se encuentran en situación de especial vulnerabilidad social, por lo que podemos decir que se trata de programas con un alcance de gran valor social. Desde un punto de vista de la eficacia de la iniciativa, se podría afirmar que ha logrado algunos de los objetivos que se planteaban como es fomentar la contratación en el ámbito local y dinamizar el mercado de trabajo durante la duración de los proyectos. No obstante, no parece tan clara la contribución del mismo respecto a la mejora de la empleabilidad futura de las personas participantes una vez finalizado el contrato estipulado, al menos no en el caso de los jóvenes. Es decir, parece que estos programas no son efectivos para el logro de la reinserción de una gran parte de los colectivos objetivo de dichas políticas. Sin embargo, sí que se observan algunos resultados positivos para desempleados mayores de 30 años con especiales dificultades de inserción. Por tanto, nuestros resultados están apuntando a la necesidad de ajustar mucho más los objetivos y, sobre todo, los colectivos destinatarios de estas medidas, para conseguir un impacto mayor sobre la empleabilidad y la inserción de los mismos.

A pesar de que los resultados presentados no permiten hablar de una ganancia clara en la empleabilidad de los colectivos tratados, hay que precisar que, para valorar de manera completa estas iniciativas, sería necesario tener en cuenta también sus efectos sobre la tasa de salida del paro a la inactividad, de cara a evaluar si logra reducir la subida de la tasa de inactividad por el efecto «trabajador desanimado». En términos generales, las PAE son muy necesarias para combatir el desempleo, que de forma tan grave afecta al mercado de trabajo español. Esto es especialmente importante para quienes están en los extremos de su carrera laboral (jóvenes y mayores de 45 años) y para los colectivos más vulnerables (bajo nivel de estudios, desempleados de larga duración, etc.). Por tanto, los ejercicios de evaluación de las PAE como el 
presentado en este artículo, deben considerarse como un instrumento de apoyo al diseño de dichas políticas para futuras convocatorias.

Finalmente, indicar que las evaluaciones cuasiexperimentales como las presentadas en este artículo son todavía escasas en España. La evaluación presentada es relativamente sencilla y de coste reducido, pues los datos ya existen y lo único que se necesita es una extracción correcta de las bases de datos disponibles y un análisis que, hoy en día, es mucho más sencillo de acometer gracias a diferentes paquetes estadísticos.

\section{Referencias bibliográficas}

Arellano, F. A. (2010). Do training programmes get the unemployed back to work? A look at the Spanish experience. Revista de Economía Aplicada, 18(53), 39-65.

https://www.researchgate.net/publication/47647401_Do_Training_Programmes_Get_ the Unemployed Back to Work A Look at the Spanish Experience

Arranz, J. M., García Serrano, C., \& Hernanz, V. (2013). Active labour market policies in Spain: A macroeconomic evaluation. International Labour Review, 152(2), 327-348. https://doi.org/10.1111/j.1564-913X.2013.00182.x

Blázquez, M., Herrarte, A., \& Sáez, F. (2019). Training and job search assistance programmes in Spain: The case of long-term unemployed. Journal of Policy Modeling, 41(2), 316-335.

https://doi.org/10.1016/j.jpolmod.2019.03.004

Caliendo, M. (2006). Microeconometric evaluation of labour market policies. Springer Science \& Business Media.

Card, D., Kluve, J., \& Weber, A. (2010). Active labour market policy evaluations: A meta-analysis. The economic journal, 120(548), F452-F477. https://doi.org/10.1111/j.1468-0297.2010.02387.x

Card, D., Kluve, J., \& Weber, A. (2018). What works? A meta analysis of recent active labor market program evaluations. Journal of the European Economic Association, 16(3), 894931. https://doi.org/10.1093/jeea/jvx028

Cueto, B., \& Mato, F. J. (2009). A nonexperimental evaluation of training programmes: regional evidence for Spain. The Annals of Regional Science, 43(2), 415-433. https://doi.org/10.1007/s00168-008-0214-2

De la Rica, S. (2015). Políticas activas de empleo: una panorámica. Fedea policy papers, 1, 1-32. https://documentos.fedea.net/pubs/fpp/2015/01/FPP2015-01.pdf

Escudero, V., Kluve, J., López Mourelo, E., \& Pignatti, C. (2019). Active labour market programmes in Latin America and the Caribbean: Evidence from a meta-analysis. The Journal of Development Studies, 55(12), 2644-2661. https://doi.org/10.1080/00220388.2018.1546843

Felgueroso, F., García-Pérez, J. I., \& Jiménez, S. (2018). Perfilado estadístico: un método para diseñar políticas activas de empleo. Editorial Centro de Estudios Ramón Areces. https://www.fundacionareces.es/recursos/doc/portal/2018/05/03/perfilado-estadistico.pdf

García-Pérez, J. I., \& Rebollo-Sanz, Y. F. (2009a). The use of Permanent Contracts Across Spanish Regions: Do regional wage subsidies work? Investigaciones Económicas, 33(1), 97-130. https://www.redalyc.org/pdf/173/17312887004.pdf 
García-Pérez, J. I., \& Rebollo-Sanz, Y. F. (2009b). Do wage subsidies affect the subsequent employment stability of permanent workers?: the case of Spain. Moneda y Crédito, 228, 65-103.

http://citeseerx.ist.psu.edu/viewdoc/download?doi=10.1.1.529.8719\&rep=rep1\&type=pdf

Gerfin, M., \& Lechner, M. (2002). A microeconometric evaluation of the active labour market policy in Switzerland. The Economic Journal, 112(482), 854-893. https://doi.org/10.1111/1468-0297.00072

Hohmeyer, K., \& Wolff, J. (2010). Direct job creation revisited. Is it effective for welfare recipients and does it matter whether participants receive a wage? IAB, Discussion Paper No. 21/2010, 1-62. http://doku.iab.de/discussionpapers/2010/dp2110.pdf

INAEM, Instituto Aragonés de Empleo. (2015). Estudio sobre la valoración económica y social de las políticas activas del Instituto Aragonés de Empleo. https://inaem.aragon.es/sites/default/files/inaem_2010-2014_25112015.pdf

Kluve, J. (2010). The effectiveness of European active labor market programs. Labour economics, 17(6), 904-918. https://doi.org/10.1016/j.labeco.2010.02.004

Kuddo, A. (2009). Employment services and active labor market programs in Eastern European and Central Asian countries. SP, Social Protection Discussion Paper No. 0918, 1-87. http://documents.worldbank.org/curated/en/801251468170040578/Employmentservices-and-active-labor-market-programs-in-Eastern-European-and-Central-Asian countries

Leuven, E., \& Sianesi, B. (2003). PSMATCH2: Stata module to perform full Mahalanobis and propensity score matching, common support graphing, and covariate imbalance testing. Statistical Software Components.

Smith, J.A., \& Todd, P. E. (2005). Does matching overcome LaLonde's critique of nonexperimental estimators? Journal of econometrics, 125(1-2), 305-353. https://doi.org/10.1016/j.jeconom.2004.04.011

Ramos, R., Suriñach, J., \& Artís, M. (2009). La efectividad de las politicas activas de mercado de trabajo para luchar contra el paro. La experiencia de Cataluña (Working Papers No. 2009/19). Research Institute of Applied Economics. http://www.ub.edu/irea/working_papers/2009/200919.pdf

Rebollo-Sanz, Y. F. (2018). El Modelo de Perfilado Estadístico: una herramienta eficiente para caracterizar a los demandantes de empleo. En F. Felgueroso, J. I. García-Pérez, \& S. Jiménez (Eds.), Perfilado estadístico: un método para diseñar políticas activas de empleo (pp. 17-93). Editorial Centro de Estudios Ramón Areces.

https://www.fundacionareces.es/recursos/doc/portal/2018/05/03/perfilado-estadistico.pdf

Roy, A. S., \& Wong, G. (2000). Direct job creation programs: Evaluation lessons on cost-effectiveness. Canadian Public Policy/Analyse de Politiques, 26(2), 157-169. https://doi.org/10.2307/3552553 